\title{
THERMOPHORESIS OF AN ARBITRARY THREE-DIMENSIONAL ARRAY OF $N$ INTERACTING ARBITRARY SPHERES
}

\author{
Huan J. Keh* and Shih H. Chen \\ Department of Chemical Engineering, National Taiwan University, Taipei 106-17 Taiwan, R.O.C.
}

(First received 25 January 1996; and in final form 6 March 1996)

\begin{abstract}
A boundary-collocation technique, used earlier by Chen and Keh [(1996) Aerosol Sci. Technol. 24, 21-35] in the study of the thermophoresis of $N$ coaxial spheres along the line of their centers, is extended to describe the motion of an assemblage of $N$ aerosol spheres arranged arbitrarily in three-dimensional space. The spheres are allowed to differ in radius, in thermal conductivity and in surface properties; they may move independently, or they may be linked by infinitesimally thin rods to form a rigid aggregate. The Knudsen numbers are assumed to be small so that the fluid flow is described by a continuum model with a thermal creep and a hydrodynamic slip at the particle surfaces.

Results are presented in terms of pair-interaction coefficients for the thermophoretic velocities of the particles. For two-sphere systems, the translational and angular velocities of the particles at all orientations and separation distances agree very well with the exact solutions obtained by using spherical bipolar coordinates or the asymptotic solutions obtained by using a method of reflections. The particle-interaction parameters of linear chains of three spheres show that the existence of the third sphere can significantly affect the mobilities of the other two spheres. For the cases of a rigid dumbbell composed of two spheres, the numerical solutions for the particle velocities compare quite favorably with the formulas derived analytically. Finally, our numerical results for the interaction between two spheres are used to find the effect of volume fractions of particles of each type on the mean thermophoretic velocities in a polydisperse aerosol. Copyright $(\mathbb{C}) 1996$ Elsevier Science Ltd.
\end{abstract}

\section{NOMENCLATURE}

$a, a_{i}$ particle radius (m)

$A, A_{j}$ thermophoretic mobility of a sphere defined by equation (1b) $\left(\mathrm{m}^{2} \mathrm{~s}^{-1} \mathrm{~K}^{-1}\right)$

$A_{j m n}, B_{j m n}, C_{j m n}$

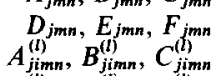

$b_{i}, c_{i}, d_{i}$

$B_{j i n}^{*}, C_{j i n}^{*}, E_{j i n}^{*}$

$B_{j i n}^{* *}, C_{j i n}^{* *}, E_{j i n}^{* *}$

$C_{\mathrm{m}}, C_{\mathrm{mi}}$

$C_{\mathrm{s}}, C_{\mathrm{si}}$

$C_{t}, C_{t i}$

$\stackrel{e}{e}$

$\underline{e}_{x}, \underline{e}_{y}, \underline{e}_{z}$

$\underline{e}_{\mathrm{r}_{i}}, \boldsymbol{e}_{\theta_{i}}, \underline{e}_{\phi_{i}}$

$\tilde{E}_{i}$

$\underset{G_{j i n}}{\stackrel{F_{i}}{E_{i}}}$

$\stackrel{I}{\tilde{k}}$

$\tilde{k}$

$k, k_{i}$

$k_{i}^{*}$

$K_{i j}^{(n)}$

1

$M, M^{*}$

$M_{i j}, \underset{\approx}{N_{i j}}$

$M_{i j}^{(p)}, \boldsymbol{M}_{i j}^{(n)}, \tilde{N}_{i j}$

coefficients defined by equation (13)

functions of $r_{i}, \theta_{i}$ and $\phi_{i}$ in equation (15)

rectangular coordinates of the center of sphere $i(\mathrm{~m})$

functions of $r_{i}, \theta_{i}$ and $\phi_{i}$ defined by equation (17)

functions of $r_{i}, \theta_{i}$ and $\phi_{i}$ defined by equation (18)

dimensionless coefficient accounting for the hydrodynamic slip

dimensionless coefficient accounting for the thermal slip

dimensionless coefficient accounting for the temperature jump

unit vector directed from the center of sphere 1 toward the center of sphere 2

unit vectors in rectangular coordinates

unit vectors in the spherical coordinates originated from particle $i$

undisturbed temperature gradient $\left(\mathrm{K} \mathrm{m}^{-1}\right)$

drag force acting on sphere $i(\mathrm{~N})$

function of $r_{i}$ and $\mu_{i}$ defined by equation (9)

unit dyadic

thermal conductivity of the fluid ( $\mathrm{W} \mathrm{m}^{-1} \mathrm{~K}^{-1}$ )

thermal conductivity of the particle $\left(\mathrm{W} \mathrm{m}^{-1} \mathrm{~K}^{-1}\right)$

ratio of thermal conductivities between sphere $i$ and the fluid

the number of collocation rings on each particle surface

dimensionless mobility parameters defined by equation (36)

mean free path of the gas molecules (m)

the number of terms reserved in the Fourier series

dimensionless mobility tensors defined by equation (22)

dimensionless mobility parameters defined by equation (31)

the number of particles in an assemblage

$P_{n}^{m} \quad$ the associated Legendre function of order $m$ and degree $n$

*Author to whom correspondence should be addressed 
$r_{0 i}$ equal to $\left|r_{0 i}\right|$, distance between the hydrodynamic center of a rigid cluster and the center of its sphere $i(\mathrm{~m})$

$r_{12}$ distance between centers of sphere 1 and sphere $2(\mathrm{~m})$

$r_{i}, \theta_{i}, \phi_{i}$ spherical coordinates measured from the origin of sphere $i$

$\underline{r}_{i}$ position vector in spherical coordinates $\left(r_{i}, \theta_{i}, \phi_{i}\right)(\mathrm{m})$

$R_{j m n}, \tilde{S_{j n n}}$ coefficients in the expression of equation (4a) for the temperature field of the fluid

$\bar{R}_{i m n}, \bar{S}_{i m n}$ coefficients in the expression of equation (4b) for the temperature field of sphere $i$

$R_{j i m n}^{\prime}, S_{j i m n}^{\prime}$ functions of $r_{i}, \theta_{i}$ and $\phi_{i}$ in equation (5)

$T$ temperature field of the fluid $(\mathrm{K})$

$T_{i} \quad$ temperature field of sphere $i(\mathrm{~K})$

$T_{x} \quad$ undisturbed temperature distribution (K)

$T_{0}$ prescribed temperature at the plane $x=0(\mathrm{~K})$

$\bar{T}, \bar{T}_{i}$ prescribed temperature at the particle center $(\mathrm{K})$

$T_{i}$ hydrodynamic torque on sphere $i$ about its center $(\mathrm{N} \mathrm{m})$

$\underline{U}_{0}, \tilde{U}_{0}$ thermophoretic velocity at the origin of a rigid cluster $\left(\mathrm{m} \mathrm{s}^{-1}\right)$

$U_{i}$ translational velocity of sphere $i\left(\mathrm{~m} \mathrm{~s}^{-1}\right)$

$U_{i x}, U_{i y}, \tilde{U}_{i z}$ components of $\mathcal{U}_{i}$ in rectangular coordinates $\left(\mathrm{m} \mathrm{s}^{-1}\right)$

$U_{j}^{(0)}, U_{j}^{(0)}$ thermophoretic velocity of sphere $j$ in the absence of all the other spheres $\left(\mathrm{m} \mathrm{s}^{-1}\right)$

$\left\langle U_{i}\right\rangle$ mean thermophoretic velocity of type $i$ particles $\left(\mathrm{m} \mathrm{s}^{-1}\right)$

2 velocity field of the fluid $\left(\mathrm{m} \mathrm{s}^{-1}\right)$

$v_{r_{i}}, v_{\theta_{i}}, v_{\phi_{i}}$ components of $\underset{\sim}{\mathrm{D}}$ in spherical coordinates $\left(r_{i}, \theta_{i}, \phi_{i}\right)\left(\mathrm{ms} \mathrm{s}^{-1}\right)$

$w$ separation parameter defined by equation (44)

$x, y, z$ rectangular coordinates $(\mathrm{m})$

Greek letters

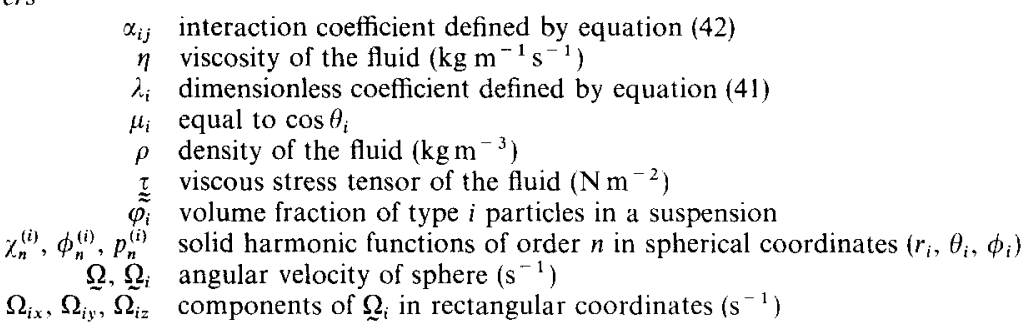

\section{INTRODUCTION}

Thermophoresis refers to the motion of aerosol particles in response to a temperature gradient. This phenomenon was first described in 1870 by Tyndall, who observed a dust-free zone in a dusty gas around a hot body (Waldmann and Schmitt, 1966). The thermophoretic effect can be explained by appealing to the kinetic theory of gases (Kennard, 1938). The higher-energy molecules in the hot region of the gas impinge on the particles with greater momenta than molecules coming from the cold region, thus leading to the migration of the particles in the direction opposite to the temperature gradient. Being a mechanism for the capture of aerosol particles on cold surfaces, thermophoresis is of considerable importance in many practical applications, such as sampling of aerosol particles (Friedlander, 1977), cleaning of air (Sasse et al., 1994), scale formation on surfaces of heat exchangers (Montassier et al., 1991), modified chemical vapor deposition (Weinberg, 1982), microelectronic manufacturing (Ye et al., 1991), and nuclear reactor safety (Williams and Loyalka, 1991).

Based on the assumptions of small Knudsen number $(l / a$, where $a$ is the radius of the particle and $l$ the mean free path of the gas molecules), small thermal Peclet number and small Reynolds number as well as the effects of temperature jump, thermal creep and hydrodynamic slip at the gas-particle surface, Brock (1962) obtained the thermophoretic velocity of an aerosol sphere in a constant temperature gradient $\nabla T_{\infty}$ as

$$
\underline{U}^{(0)}=-A \nabla T_{\infty},
$$

where the thermophoretic mobility

$$
A=\left[\frac{2 C_{\mathrm{s}}\left(k+\hat{k} C_{\mathrm{t}} l / a\right)}{\left(1+2 C_{\mathrm{m}} l / a\right)\left(2 k+\hat{k}+2 k C_{\mathrm{t}} l / a\right)}\right] \frac{\eta}{\rho \bar{T}} .
$$


In equation ( $1 \mathrm{~b}), \rho, \eta$ and $k$ are the density, viscosity and thermal conductivity, respectively, of the gas; $\hat{k}$ is the thermal conductivity of the particle; $\bar{T}$ is the bulk-gas absolute temperature at the particle center in the absence of the particle (or the mean gas temperature in the vicinity of the particle); $C_{\mathrm{s}}, C_{\mathrm{t}}$ and $C_{\mathrm{m}}$ are dimensionless coefficients accounting for the thermal creep, temperature jump and hydrodynamic slip phenomena, respectively, at the particle surface and must be determined experimentally for each gas-solid system. A set of reasonable kinetic-theory values for complete thermal and momentum accommodations appear to be $C_{\mathrm{s}}=1.17, C_{\mathrm{t}}=2.18$ and $C_{\mathrm{m}}=1.14$ (Talbot et al., 1980). Note that the negative sign in equation (1a) indicates that the particle migration is in the direction of decreasing temperature and $\rho \bar{T}$ in equation (1b) is a constant for an ideal gas at constant pressure. Brock's (1962) analysis was extended to spheroidal particles by using prolate and oblate spheroidal coordinate systems (Leong, 1984).

In most real situations of thermophoresis, aerosol particles are not isolated and will move in the presence of neighboring particles. Through an exact representation in spherical bipolar coordinates, the thermophoretic motion of two separate, arbitrary spherical particles along the line of their centers was recently examined (Chen and Keh, 1995). Numerical results of correction to equation (1b) for each particle were presented for various cases. On the other hand, the thermophoresis of two arbitrary spheres whose connecting axis is oriented arbitrarily relative to the temperature gradient was analyzed using a method of reflections (Keh and Chen, 1995). The particle velocities were determined in an approximate solution of increasing powers of $r_{12}^{-1}$ up to $\mathrm{O}\left(r_{12}^{-7}\right)$, where $r_{12}$ is the center-to-center distance between the particles. Several important conclusions result from these investigations of the two-sphere interactions in thermophoresis. First, the particle interaction effects on thermophoresis in general are much weaker than on sedimentation, because the disturbance to the fluid velocity field caused by a thermophoretic sphere decays faster (as $r^{-3}$, where $r$ is the distance from the particle center) than that caused by a settling sphere (as $r^{-1}$ ). In sedimentation, there is a net gravitational force exerted on the particle and this force is balanced by a nonzero hydrodynamic force. In thermophoresis, however, there is no hydrodynamic force exerted on the particle. As a consequence, the disturbance velocity fields in the surrounding fluid for the two situations decay at different rates with $r$. Second, for the situation of two identical spheres aligned parallel to the prescribed temperature gradient, the interaction effects make each particle move faster than the velocity it would possess if isolated, while for the situation of two identical spheres undergoing thermophoresis normal to the line of their centers, each particle migrates slower than its undisturbed velocity. Third, the thermophoretic velocity of each of two coexistent identical spheres, which can be arbitrarily oriented, is unaffected by the presence of the other in the case of $k / k=0$ or $C_{\mathrm{t}} l / a \rightarrow \infty$. Fourth, the influence of the interactions between the particles in general is far greater on the smaller one than on the larger one.

In view of the fact that the interactions among multiple particles may be important and aggregates of particles can be formed in concentrated aerosol suspensions, in a previous article (Chen and Keh, 1996) we studied the axisymmetric thermophoretic motion of a string of $N$ freely suspended or linked spherical particles along the line of their centers using a boundary collocation technique. In that analysis, the particles could differ in physical properties and in radius and they were allowed to be unequally spaced. The numerical results of particle interaction effects could be obtained with good accuracy even when the particles were touching one another.

This paper is an extension of the previous work (Chen and Keh, 1996) to the situation of the thermophoretic motion of multiple spheres in an arbitrary configuration. Again, the spheres may differ in radius and in physical properties. The quasi-steady energy and momentum equations applicable to the system are solved by using the boundary collocation technique and the particle interaction parameters are obtained for various cases. For the simple case of thermophoresis of two spheres normal to the line of their centers, our numerical results for the particle velocities show excellent agreement with the asymptotic solution obtained by using the method of reflections. The combined analytical-numerical solution method for the thermophoresis of freely suspended spheres is also employed to 
examine the thermophoretic motion of a three-dimensional rigid cluster composed of $N$ arbitrary spheres connected by thin rods of arbitrary lengths through their centers. The complete collocation results for the interaction effects between pairs of spheres are also used to evaluate the mean thermophoretic velocity in a bounded suspension of aerosol spheres.

\section{ANALYSIS FOR MULTIPLE SPHERES}

Consider the thermophoretic motion of $N$ spherical particles in an infinite gaseous medium, which is assumed to be Newtonian and incompressible, in an arbitrary threedimensional configuration as shown in Fig. 1. For convenience, the Cartesian coordinate system $(x, y, z)$ is established such that the center of the first sphere is at the origin and the uniform imposed thermal gradient $\nabla T_{\infty}$ equals $E_{\infty} e_{x}$, where $e_{x}$ together with $\underline{e}_{y}$ and $e_{z}$ are the unit vectors in the coordinate system. The position of the center of particle $i$ is represented by coordinates $\left(b_{i}, c_{i}, d_{i}\right)$, and we have set $b_{1}=c_{1}=d_{1}=0$. The particles may be formed from different materials and have unequal radii. Our purpose here is to determine the correction to equation (1) for the motion of each particle due to the presence of the other ones in proximity. At first, the temperature distributions inside and outside the particles and the fluid velocity field must be solved.

\subsection{Temperature distributions}

The thermophoretic motion of multiple particles is inherently unsteady. However, the problem can be considered quasi-steady if the Reynolds and Peclet numbers are small. The conduction equation governing the temperature distribution $T(r)$ for the suspending fluid of constant thermal conductivity $k$ is

$$
\nabla^{2} T=0
$$

For the temperature field $T_{i}(r)$ inside the particle $i$, one has

$$
\nabla^{2} T_{i}=0, \quad i=1,2, \ldots, N
$$

The boundary conditions at the particle surfaces require that the normal heat fluxes be continuous and a temperature jump occur which is proportional to the normal temperature gradient (Kennard, 1938). Also, the fluid temperature must approach the linear prescribed field far from the particles and the temperature inside each particle is finite everywhere. Thus, one has

$$
\begin{aligned}
r_{i}=a_{i}: & T-T_{i}=C_{\mathrm{t} i} l \frac{\partial T}{\partial r_{i}}, \\
& k \frac{\partial T}{\partial r_{i}}=k_{i} \frac{\partial T_{i}}{\partial r_{i}}, \\
r_{i}<a_{i}: & T_{i} \text { is finite, } \\
\left(x^{2}+y^{2}+z^{2}\right)^{1 / 2} \rightarrow \infty: \quad & T \rightarrow T_{\infty}=T_{0}+E_{\infty} x,
\end{aligned}
$$

for $i=1,2, \ldots, N$. Here, $a_{i}, k_{i}$ and $C_{t i}$ are the radius, thermal conductivity and temperature jump coefficient of particle $i$ respectively, $l$ is the mean free path of the surrounding fluid, $\left(r_{i}, \theta_{i}, \phi_{i}\right)$ are spherical coordinates measured from the center of particle $i$, and $T_{0}$ is the temperature far from the particles at the plane $x=0$.

The fundamental solution of Laplace's equation that is capable of describing an arbitrary disturbance on the surface of a sphere consists of the internal and external solid spherical harmonic functions. For $N$ spherical particles dispersed in the temperature field, the general 


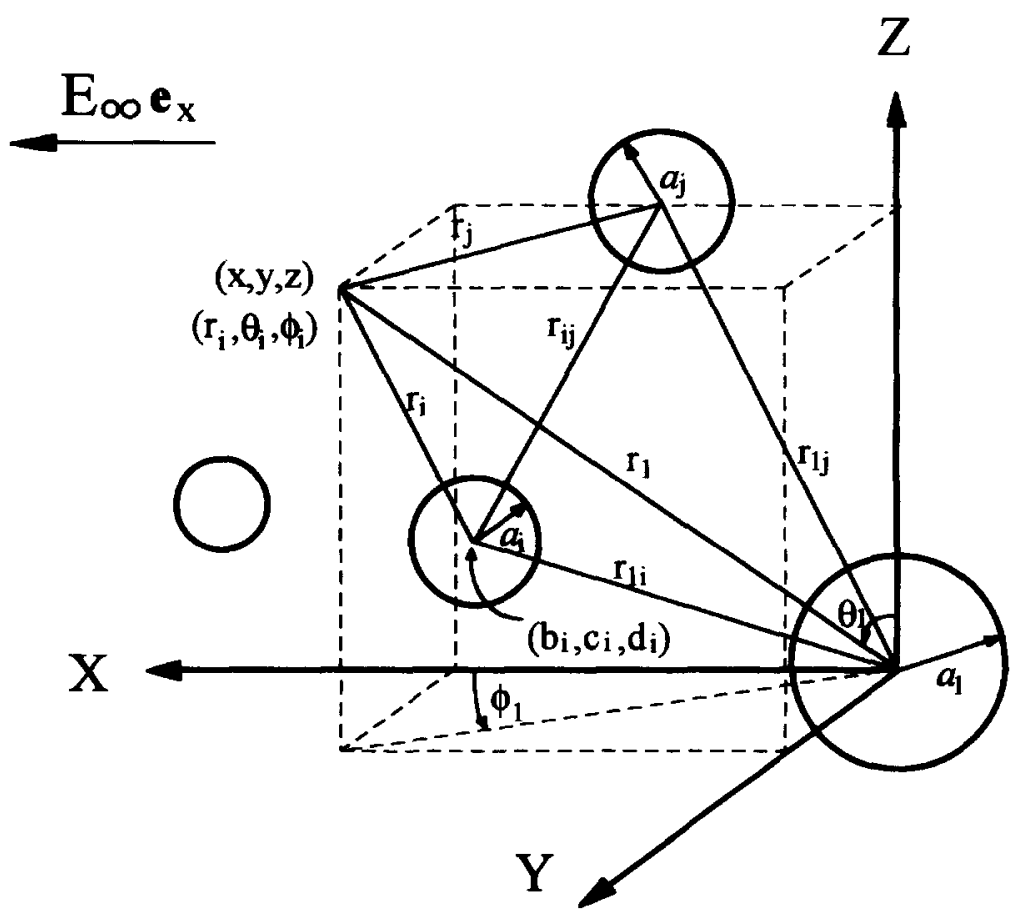

Fig. 1. Geometrical sketch for the thermophoresis of multiple aerosol spheres.

solution to equation (2) can be written as

$$
\begin{aligned}
T= & E_{\infty} \sum_{j=1}^{N} \sum_{n=0}^{\infty} \sum_{m=0}^{n} r_{j}^{-n-1} P_{n}^{m}\left(\mu_{j}\right)\left[R_{j m n} \cos \left(m \phi_{j}\right)+S_{j m n} \sin \left(m \phi_{j}\right)\right] \\
+ & E_{\infty} x+T_{0} \\
T_{i}= & E_{\infty} \sum_{n=0}^{\infty} \sum_{m=0}^{n} r_{i}^{n} P_{n}^{m}\left(\mu_{i}\right)\left[\bar{R}_{i m n} \cos \left(m \phi_{i}\right)+\bar{S}_{i m n} \sin \left(m \phi_{i}\right)\right] \\
& +E_{\infty} x+T_{0}, \quad i=1,2, \ldots, N .
\end{aligned}
$$

Here $P_{n}^{m}$ are the associated Legendre functions and $\mu_{i}$ is used to denote $\cos \theta_{i}$ for brevity. A solution of the form of equation (4) immediately satisfies boundary conditions (3c) and (3d). The unknown coefficients $R_{j m n}, S_{j m n}, \bar{R}_{i m n}$ and $\bar{S}_{i m n}$ are to be determined using the boundary conditions at the particle surfaces. It is understood that $S_{j m n}=\bar{S}_{i m n}=0$ if $m=0$.

Application of the boundary conditions (3a) and (3b) along the surface of each sphere to equation (4) leads to

$$
\begin{aligned}
& \sum_{j=1}^{N} \sum_{n=0}^{\infty} \sum_{m=0}^{n}\left\{R_{j m n}\left[r_{j}^{-n-1} P_{n}^{m}\left(\mu_{j}\right) \cos \left(m \phi_{j}\right)\right]_{r_{i}=a_{i}}+S_{j m n}\left[r_{j}^{-n-1} P_{n}^{m}\left(\mu_{j}\right) \sin \left(m \phi_{j}\right)\right]_{r_{i}=a_{i}}\right\} \\
& \quad-\sum_{n=0}^{\infty} \sum_{m=0}^{n}\left(a_{i}^{n}+n a_{i}^{n-1} k_{i}^{*} C_{t i} l\right) P_{n}^{m}\left(\mu_{i}\right)\left[\bar{R}_{i m n} \cos \left(m \phi_{i}\right)+\bar{S}_{i m n} \sin \left(m \phi_{i}\right)\right] \\
& \quad=k_{i}^{*} C_{t i} l\left(1-\mu_{i}^{2}\right)^{1 / 2} \cos \phi_{i}, \\
& \sum_{j=1}^{N} \sum_{n=0}^{\infty} \sum_{m=0}^{n}\left[R_{j m n} R_{j i m n}^{\prime}\left(a_{i}, \mu_{i}, \phi_{i}\right)+S_{j m n} S_{j i m n}^{\prime}\left(a_{i}, \mu_{i}, \phi_{i}\right)\right] \\
& -k_{i}^{*} \sum_{n=0}^{\infty} \sum_{m=0}^{n} n a_{i}^{n-1} P_{n}^{m}\left(\mu_{i}\right)\left[\bar{R}_{i m n} \cos \left(m \phi_{i}\right)+\bar{S}_{i m n} \sin \left(m \phi_{i}\right)\right] \\
& =\left(k_{i}^{*}-1\right)\left(1-\mu_{i}^{2}\right)^{1 / 2} \cos \phi_{i},
\end{aligned}
$$


where $k_{i}^{*}=k_{i} / k$, functions $R_{j i m n}^{\prime}\left(r_{i}, \mu_{i}, \phi_{i}\right)$ and $S_{j i m n}^{\prime}\left(r_{i}, \mu_{i}, \phi_{i}\right)$ are defined by equation (2.7) of Keh and Yang (1991) and $i=1,2, \ldots, N$. In equation (5a), the coordinates $\left(r_{j}, \theta_{j}, \phi_{j}\right)$ of an arbitrary position relative to the center of the $j$ th particle are related to the coordinates $\left(r_{i}, \theta_{i}, \phi_{i}\right)$ of the position relative to the center of the $i$ th sphere by equation (2.8) of Keh and Yang (1991). To satisfy the boundary conditions (5a) and (5b) exactly along the entire surface of each particle would require the solution of the entire infinite arrays of unknown constants $R_{j m n}, S_{j m n}, \bar{R}_{i m n}$ and $\bar{S}_{i m n}$. However, the boundary collocation method allows one to truncate the infinite series in equation (4) and then to enforce the boundary conditions at a finite number of discrete points on the surface of each sphere (Hassonjee et al., 1988; Keh and Yang, 1991; Williams and Loyalka, 1991).

To apply the collocation technique, the order of summation $\sum_{n=0}^{\infty} \sum_{m=0}^{n}$ in equations (4) and (5) is changed to $\sum_{m=0}^{\infty} \sum_{n=m}^{\infty}$ without the loss of any terms in the series. Then the infinite series $\sum_{m=0}^{\infty}$ is truncated after the first $M$ terms and the infinite series $\sum_{n=m}^{\infty}$ is truncated after its first $K$ terms for each value of $j$ or $i$. With this arrangement, equation (4) becomes

$$
\begin{aligned}
T= & E_{\infty} \sum_{j=1}^{N} \sum_{m=0}^{M-1} \sum_{n=m}^{m+K-1} r_{j}^{-n-1} P_{n}^{m}\left(\mu_{j}\right)\left[R_{j m n} \cos \left(m \phi_{j}\right)+S_{j m n} \sin \left(m \phi_{j}\right)\right] \\
& +E_{\infty} x+T_{0}, \\
T_{i}= & E_{\infty} \sum_{m=0}^{M-1} \sum_{n=m}^{m+K-1} r_{i}^{n} P_{n}^{m}\left(\mu_{i}\right)\left[\bar{R}_{i m n} \cos \left(m \phi_{i}\right)+\bar{S}_{i m n} \sin \left(m \phi_{i}\right)\right] \\
& +E_{\infty} x+T_{0}, \quad i=1,2, \ldots, N,
\end{aligned}
$$

and equation (5) must be altered accordingly.

Equation (6) leaves a total of $2 N K(2 M-1)$ unknown coefficients $R_{j m n}, S_{j m n}, \bar{R}_{i m n}$ and $\bar{S}_{i m n}\left(S_{j 0 n}=\bar{S}_{i 0 n}=0\right)$ to be determined. To generate the equations needed to evaluate these unknown constants, we multiply the truncated form of equation (5) by the function sets $\cos \left(m^{\prime} \phi_{i}\right)$ and $\sin \left(m^{\prime} \phi_{i}\right)$, integrate with respect to $\phi_{i}$ from 0 to $2 \pi$, and utilize the orthogonality properties of these functions in this interval to obtain

$$
\begin{aligned}
& \sum_{j=1}^{N} \sum_{m=0}^{M-1} \sum_{n=m}^{m+K-1}\left\{R_{j m n} \int_{0}^{2 \pi}\left[r_{j}^{-n-1} P_{n}^{m}\left(\mu_{j}\right) \cos \left(m \phi_{j}\right)\right]_{r_{i}=a_{i}} \cos \left(m^{\prime} \phi_{i}\right) \mathrm{d} \phi_{i}\right. \\
& \left.+S_{j m n} \int_{0}^{2 \pi}\left[r_{j}^{-n-1} P_{n}^{m}\left(\mu_{j}\right) \sin \left(m \phi_{j}\right)\right]_{r_{i}=a_{i}} \cos \left(m^{\prime} \phi_{i}\right) \mathrm{d} \phi_{i}\right\} \\
& -\pi \sum_{n=m^{\prime}}^{m^{\prime}+K-1}\left(a_{i}^{n}+n a_{i}^{n-1} k_{i}^{*} C_{t i} l\right) \bar{R}_{i m^{\prime} n} P_{n}^{m^{\prime}}\left(\mu_{i}\right) \\
& =\left[\begin{array}{ll}
\pi \sum_{n=0}^{K-1}\left(a_{i}^{n}+n a_{i}^{n-1} k_{i}^{*} C_{\mathrm{ti}} l\right) \bar{R}_{i 0 n} P_{n}^{0}\left(\mu_{i}\right) & \left(m^{\prime}=0\right) \\
\pi k_{i}^{*} C_{\mathrm{t} i} l\left(1-\mu_{i}^{2}\right)^{1 / 2} & \left(m^{\prime}=1\right) \\
0 & \left(m^{\prime}=2,3, \ldots, M-1\right),
\end{array}\right. \\
& \sum_{j=1}^{N} \sum_{m=0}^{M-1} \sum_{n=m}^{m+K-1}\left\{R_{j m n} \int_{0}^{2 \pi}\left[r_{j}^{-n-1} P_{n}^{m}\left(\mu_{j}\right) \cos \left(m \phi_{j}\right)\right]_{r_{i}=a_{i}} \sin \left(m^{\prime} \phi_{i}\right) \mathrm{d} \phi_{i}\right. \\
& \left.+S_{j m n} \int_{0}^{2 \pi}\left[r_{j}^{-n-1} P_{n}^{m}\left(\mu_{j}\right) \sin \left(m \phi_{j}\right)\right]_{r_{i}=a_{i}} \sin \left(m^{\prime} \phi_{i}\right) \mathrm{d} \phi_{i}\right\} \\
& -\pi \sum_{n=m^{\prime}}^{m^{\prime}+K-1}\left(a_{i}^{n}+n a_{i}^{n-1} k_{i}^{*} C_{\mathrm{ti}} l\right) \bar{S}_{i m^{\prime} n} P_{n}^{m^{\prime}}\left(\mu_{i}\right) \\
& =0 \quad\left(m^{\prime}=1,2, \ldots, M-1\right)
\end{aligned}
$$




$$
\begin{aligned}
\sum_{j=1}^{N} \sum_{m=0}^{M-1} \sum_{n=m}^{m+K-1}\left\{R_{j m n} \int_{0}^{2 \pi} R_{j i m n}^{\prime}\left(a_{i}, \mu_{i}, \phi_{i}\right) \cos \left(m^{\prime} \phi_{i}\right) \mathrm{d} \phi_{i}\right. \\
\left.+S_{j m n} \int_{0}^{2 \pi} S_{j i m n}^{\prime}\left(a_{i}, \mu_{i}, \phi_{i}\right) \cos \left(m^{\prime} \phi_{i}\right) \mathrm{d} \phi_{i}\right\} \\
-\pi k_{i}^{*} \sum_{n=m^{\prime}}^{m^{\prime}+K-1} n a_{i}^{n-1} \bar{R}_{i m^{\prime} n} P_{n}^{m^{\prime}}\left(\mu_{i}\right) \\
=\left[\begin{array}{lc}
\pi k_{i}^{*} \sum_{n=0}^{K-1} n a_{i}^{n-1} \bar{R}_{i 0 n} P_{n}^{o}\left(\mu_{i}\right) & \left(m^{\prime}=0\right) \\
\pi\left(k_{i}^{*}-1\right)\left(1-\mu_{i}^{2}\right)^{1 / 2} & \left(m^{\prime}=1\right) \\
0 & \left(m^{\prime}=2,3, \ldots, M-1\right),
\end{array}\right.
\end{aligned}
$$

$$
\begin{aligned}
\sum_{j=1}^{N} \sum_{m=0}^{M-1} \sum_{n=m}^{m+K-1}\left\{R_{j m n} \int_{0}^{2 \pi} R_{j i m n}^{\prime}\left(a_{i}, \mu_{i}, \phi_{i}\right) \sin \left(m^{\prime} \phi_{i}\right) \mathrm{d} \phi_{i}\right. \\
\left.+S_{j m n} \int_{0}^{2 \pi} S_{j i m n}^{\prime}\left(a_{i}, \mu_{i}, \phi_{i}\right) \sin \left(m^{\prime} \phi_{i}\right) \mathrm{d} \phi_{i}\right\} \\
-\pi k_{i}^{*} \sum_{n=m^{\prime}}^{m^{\prime}+K-1} n a_{i}^{n-1} \bar{S}_{i m^{\prime} n} P_{n}^{m^{\prime}}\left(\mu_{i}\right) \\
=0 \quad\left(m^{\prime}=1,2, \ldots, M-1\right)
\end{aligned}
$$

where $i=1,2, \ldots, N$. The above boundary conditions are satisfied at $K$ discrete values of $\theta_{i}$ (rings) at the surface of each particle $i$. This results in a set of $2 N K(2 M-1)$ simultaneous linear algebraic equations, which is exactly equal in number to the unknown constants $R_{j m n}$, $S_{j m n}, \bar{R}_{i m n}$ and $\bar{S}_{i m n}$ in the truncated solution (6) for the temperature field. These $2 N K(2 M-1)$ equations can be solved using any standard matrix-reduction technique. Note that the definite integrals in equation (7) for each collocation ring must be performed numerically.

The accuracy of this boundary-collocation, truncated-series solution technique can be improved to any degree by taking sufficiently large values of $M$ and $K$. Naturally, the truncation error reduces to zero as $M \rightarrow \infty$ and $K \rightarrow \infty$. In general cases, the series in equation (6) converge quite rapidly, and very good accuracy can be achieved with only a small number of terms in the Fourier series $(M)$ and collocation rings on each particle $(K)$.

One special case of the general three-dimensional theory described above is the case with planar symmetry, i.e. the centers of all the spheres lie in the plane $y=0$. For these planar symmetric configurations, the constants $S_{j m n}$ and $\bar{S}_{i m n}$ are all zero and equations (7b) and (7d) become trivial. So the number of unknowns $\left(R_{j m n}\right.$ and $\bar{R}_{i m n}$ only) is reduced to $2 N K M$ and they can be determined by an equal number of equations in the form of equations ( $7 \mathrm{a})$ and (7c) satisfied at $K$ discrete rings on the surface of each sphere. Furthermore, there are two special cases which can be deduced from the planar case: a string of spheres oriented parallel to the prescribed thermal gradient (the centers of all spheres lie on $x$-axis) and a string of spheres oriented normal to the applied field (the centers of all spheres lie along the $z$-axis). The temperature distributions for the former case are axisymmetric about the $x$-axis and were solved in a previous article (Chen and Keh, 1996).

For the configuration of a finite chain of spheres located on the $z$-axis, we have $\phi_{i}=\phi$, $b_{i}=c_{i}=0(i=1,2, \ldots, N)$, and only the coefficients for $m=1$ will be nonzero. Thus, the 
truncated form of equation (5) can be simplified as

$$
\sum_{j=1}^{N} \sum_{n=1}^{K} R_{j 1 n}\left[r_{j}^{-n-1} P_{n}^{1}\left(\mu_{j}\right)\right]_{r_{i}=a_{i}}-\sum_{n=1}^{K}\left(a_{i}^{n}+n a_{i}^{n-1} k_{i}^{*} C_{\mathrm{ti}} l\right) \bar{R}_{i 1 n} P_{n}^{1}\left(\mu_{i}\right)=k_{i}^{*} C_{\mathrm{t} i} l\left(1-\mu_{i}^{2}\right)^{1 / 2}
$$

$$
\sum_{j=1}^{N} \sum_{n=1}^{K} R_{j 1 n} G_{j i n}\left(a_{i}, \mu_{i}\right)-k_{i}^{*} \sum_{n=1}^{K} n a_{i}^{n-1} \vec{R}_{i 1 n} P_{n}^{1}\left(\mu_{i}\right)=\left(k_{i}^{*}-1\right)\left(1-\mu_{i}^{2}\right)^{1 / 2}
$$

where

$$
G_{j i n}\left(r_{i}, \mu_{i}\right)=r_{j}^{-n-4}\left\{-(n+1)\left[r_{i}+\mu_{i}\left(d_{i}-d_{j}\right)\right] r_{j} P_{n}^{1}\left(\mu_{j}\right)-\left(1-\mu_{i}^{2}\right)\left(d_{i}-d_{j}\right) r_{i} \frac{\mathrm{d} P_{n}^{1}\left(\mu_{j}\right)}{\mathrm{d} \mu_{j}}\right\},
$$

and the dependence on $\phi$ factors out. Instead of using equation (7) one can apply equation (8) at $K$ discrete values of $\theta_{i}$ along the surface of each of the $N$ particles and the numerical integration with respect to the variable $\phi$ is not needed. This generates a set of $2 N K$ linear algebraic equations for the $2 N K$ unknown coefficients $R_{j 1 n}$ and $\bar{R}_{i 1 n}$. Once these coefficients are determined by a matrix-reduction technique, the temperature distributions for this special case are completely solved.

\subsection{Fluid velocity distribution}

With knowledge of the solution for the temperature field, we can now proceed to find the fluid velocity distribution. Due to the low Reynolds numbers encountered in thermophoretic motion, the fluid velocity is governed by the Stokes equations

$$
\begin{gathered}
\eta \nabla^{2} \underline{v}-\nabla p=\underline{0}, \\
\nabla \cdot \underline{v}=0,
\end{gathered}
$$

where $v(v)$ is the velocity field for the fluid flow and $p(r)$ is the corresponding pressure distribution. Owing to the thermal creep velocity and the frictional slip velocity along the particle surfaces as well as the fluid at rest far from the particles, the boundary conditions for the fluid velocity are

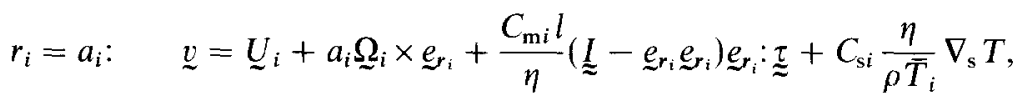

$$
\begin{aligned}
& \left(x^{2}+y^{2}+z^{2}\right)^{1 / 2} \rightarrow \infty: \quad \underset{v}{v} \rightarrow \underset{\sim}{0},
\end{aligned}
$$

for $i=1,2, \ldots, N$. Here, $\bar{T}_{i}=T_{0}+E_{\infty} b_{i}$, which is the prescribed temperature at the position of the center of particle $i \underset{\sim}{\tau}\left(=\eta\left[\nabla \underline{v}+(\nabla \underline{v})^{\mathrm{T}}\right]\right)$ is the viscous stress tensor for the fluid; $e_{r_{i}}$ is the radial unit vector in the spherical coordinates measured from the center of particle $i ; \underset{\sim}{I}$ is the unit dyadic; $C_{\mathrm{m} i}$ and $C_{\mathrm{s} i}$ are the hydrodynamic slip and thermal slip coefficients, respectively, about the surface of particle $i ; U_{i}\left(=U_{i x} e_{x}+U_{i y} e_{y}+U_{i z} e_{z}\right)$ and $\Omega_{i}\left(=\Omega_{i x} e_{x}+\Omega_{i y} e_{y}+\Omega_{i z} e_{z}\right)$ are the translational and angular velocities of particle $i$ to be determined. The tangential temperature gradient, $\nabla_{\mathrm{s}} T=\left(\underset{\sim}{I}-\underline{e}_{r_{i}}{\underline{r_{i}}}_{i}\right) \cdot \nabla T$, can be obtained from equation (6a) with coefficients determined from equation (7). The validity of the expression for the thermal creep velocity in equation (11a) is based on the assumption that the fluid is only slightly nonuniform in the undisturbed temperature on the length scale of the particle radii.

The general solution to equation $(10)$ is

$$
\underline{v}=\sum_{j=1}^{N} \sum_{n=1}^{\infty}\left[\nabla \times\left(\underline{r}_{j} \chi_{-n-1}^{(j)}\right)+\nabla \phi_{-n-1}^{(j)}-\frac{n-2}{2 \eta n(2 n-1)} r_{j}^{2} \nabla p_{-n-1}^{(j)}+\frac{n+1}{\eta n(2 n-1)} \underline{r}_{j} p_{-n-1}^{(j)}\right],
$$


where $\chi_{-n-1}^{(j)}, \phi_{-n-1}^{(j)}$ and $p_{-n-1}^{(j)}$ are the solid spherical harmonic functions of order $-(n+1)$ which depend on the spherical coordinates $\left(r_{j}, \theta_{j}, \phi_{j}\right)$ originating at the center of particle $j$ and $\underline{r}_{j}=r_{j} e_{j}$. These functions can be expressed as

$$
\left[\begin{array}{l}
\chi_{-n-1}^{(j)} \\
\phi_{-n-1}^{(j)} \\
p_{-n-1}^{(j)}
\end{array}\right]=r_{j}^{-n-1} \sum_{m=0}^{n} P_{n}^{m}\left(\mu_{j}\right)\left(\left[\begin{array}{l}
A_{j m n} \\
C_{j m n} \\
E_{j m n}
\end{array}\right] \cos \left(m \phi_{j}\right)+\left[\begin{array}{c}
B_{j m n} \\
D_{j m n} \\
F_{j m n}
\end{array}\right] \sin \left(m \phi_{j}\right)\right\}
$$

The boundary condition (11b) is immediately satisfied by a solution of this form and the unknown coefficients $A_{j m n}, B_{j m n}, \ldots$, and $F_{j m n}$ remain to be determined from equation (11a). In the construction of solution (12), the superposition of Lamb's general solution (Happel and Brenner, 1983) to equation (10) as written from $N$ different origins can be utilized owing to the linearity of the governing equations.

Substituting equation (13) into equation (12) and applying equations (2.8), (2.23) and (2.24) of Keh and Yang (1991) for the coordinate transformation leads to an expression for the fluid velocity field in terms of spherical coordinates measured from the center of the $i$ th particle:

$$
\underset{v}{v}=v_{r_{i}}\left(r_{i}, \theta_{i}, \phi_{i}\right){\underset{\sim}{r_{i}}}_{i}+v_{\theta_{i}}\left(r_{i}, \theta_{i}, \phi_{i}\right){\underset{\sim}{\theta_{i}}}_{\theta_{i}}+v_{\phi_{i}}\left(r_{i}, \theta_{i}, \phi_{i}\right){\underset{\sim}{\phi_{i}}}_{\phi_{i}},
$$

where

$$
\left[\begin{array}{l}
v_{r_{i}} \\
v_{\theta_{i}} \\
v_{\phi_{i}}
\end{array}\right]=\sum_{j=1}^{N} \sum_{n=1}^{\infty} \sum_{m=0}^{n}\left\{A_{j m n}\left[\begin{array}{l}
A_{j i m n}^{(1)} \\
A_{j i m n}^{(2)} \\
A_{j i m n}^{(3)}
\end{array}\right]+B_{j m n}\left[\begin{array}{l}
B_{j i m n}^{(1)} \\
B_{j i m n}^{(2)} \\
B_{j i m n}^{(3)}
\end{array}\right]+\cdots+F_{j m n}\left[\begin{array}{l}
F_{j i m n}^{(1)} \\
F_{j i m n}^{(2)} \\
F_{j i m n}^{(3)}
\end{array}\right]\right\},
$$

and $i=1,2, \ldots, N$. The functions $A_{j i m n}^{(l)}\left(r_{i}, \mu_{i}, \phi_{i}\right), B_{j i m n}^{(l)}\left(r_{i}, \mu_{i}, \phi_{i}\right), \ldots$, and $F_{j i m n}^{(l)}\left(r_{i}, \mu_{i}, \phi_{i}\right)$ with $l=1,2$ and 3 in equation (15) are defined by equation (2.27) of Keh and Yang (1991).

Application of boundary condition (11a) to equation (14) can be accomplished by utilizing the collocation technique presented in the previous subsection for the solution of the temperature distributions. First, the order of summation $\sum_{n=1}^{\infty} \sum_{m=0}^{n}$ in equation (15) is changed to $\sum_{m=0}^{\infty} \sum_{n=m, n \neq 0}^{\infty}$ without loss of any terms in the series. Then the infinite series $\sum_{m=0}^{\infty}$ and $\sum_{n=m, n \neq 0}^{\infty}$ are truncated after $M^{*}$ and $K^{*}$ terms, respectively, for each value of $j$. Substitution of equations (14) and (6a) after this arrangement into equation (11a) yields three algebraic formulas which leave a total of $3 N K^{*}\left(2 M^{*}-1\right)$ unknown constants $A_{j m n}$, $B_{j m n}, \ldots$, and $F_{j m n}$ to be determined (equation (13) gives $B_{j 0 n}=D_{j 0 n}=F_{j 0 n}=0$ ). Multiplying these formulas by the function sets $\cos \left(m^{\prime} \phi_{i}\right)\left(m^{\prime}=0,1,2, \ldots, M^{*}-1\right)$ and $\sin \left(m^{\prime} \phi_{i}\right)$ $\left(m^{\prime}=1,2, \ldots, M^{*}-1\right)$, integrating with respect to $\phi_{i}$ from 0 to $2 \pi$, and utilizing the orthogonality properties of these functions in this interval allow one to obtain $3\left(2 M^{*}-1\right)$ equations which are similar in form to equation (7). These equations can be satisfied at $K^{*}$ discrete values of $\theta_{i}$ (rings) along the surface of each of the $N$ particles to result in a set of $3 N K^{*}\left(2 M^{*}-1\right)$ linear algebraic equations, which can be solved by a matrixreduction method to obtain the equal-number unknown constants in terms of the particle velocities ${\underset{\sim}{i}}_{i}$ and $\Omega_{i}$. Once these constants are determined, the fluid velocity field is completely solved.

For the case of planar symmetry, i.e. the centers of all the spheres lie in the plane $y=0, U_{i y}=\Omega_{i x}=\Omega_{i z}=0$, the coefficients $A_{j m n}, D_{j m n}$ and $F_{j m n}$ are all zero and the integration of three algebraic formulas after multiplication by $\sin \left(m^{\prime} \phi_{i}\right)$ with respect to $\phi_{i}$ from 0 to $2 \pi$ is trivial. Thus, the number of unknown coefficients $\left(B_{j m n}, C_{j m n}\right.$ and $\left.E_{j m n}\right)$ is reduced to $3 N K^{*} M^{*}$ and they are determined by an equal number of collocation equations. 
Similar to the previous subsection, two special cases can be deduced from the planar case. The fluid velocity field about a chain of spheres undergoing thermophoretic motion along the line of their centers ( $x$-axis) is axisymmetric and was solved by employing the Stokes stream function (Chen and Keh, 1996). For the case of a string of spheres oriented normal to the prescribed thermal gradient (the centers of all the spheres lie on the $z$-axis), one has $\phi_{i}=\phi, b_{i}=c_{i}=0$ and $U_{i y}=U_{i z}=\Omega_{i x}=\Omega_{i z}=0(i=1,2, \ldots, N)$, and only the coefficients for $m=1$ will be nonzero. Thus, the three algebraic formulas obtained by substituting equations (14) (after truncation) and (6a) into equation (11a) can be simplified to

$$
\begin{aligned}
& \sum_{j=1}^{N} \sum_{n=1}^{K^{*}}\left[B_{j 1 n} B_{j i 1 n}^{(1)}\left(a_{i}, \mu_{i}, \phi\right)+C_{j 1 n} C_{j i 1 n}^{(1)}\left(a_{i}, \mu_{i}, \phi\right)+E_{j 1 n} E_{j i 1 n}^{(1)}\left(a_{i}, \mu_{i}, \phi\right)\right] \\
& =U_{i x}\left(1-\mu_{i}^{2}\right)^{1 / 2} \cos \phi \\
& \sum_{j=1}^{N} \sum_{n=1}^{K^{*}}\left[B_{j 1 n} B_{j i n}^{*}\left(a_{i}, \mu_{i}, \phi\right)+C_{j 1 n} C_{j i n}^{*}\left(a_{i}, \mu_{i}, \phi\right)+E_{j 1 n} E_{j i n}^{*}\left(a_{i}, \mu_{i}, \phi\right)\right] \\
& =\left(U_{i x} \mu_{i}+a_{i} \Omega_{i y}\right) \cos \phi \\
& \quad-C_{\mathrm{si}} \frac{\eta E_{\infty}}{\rho \bar{T}_{i}} \frac{1}{a_{i}}\left[\sum_{n=1}^{K} \bar{R}_{i 1 n}\left(a_{i}^{n}+n a_{i}^{n-1} k_{i}^{*} C_{i i} l\right)\left(1-\mu_{i}^{2}\right)^{1 / 2} \frac{\mathrm{d} P_{n}^{1}\left(\mu_{i}\right)}{\mathrm{d} \mu_{i}}\right. \\
& \left.-\left(a_{i}+k_{i}^{*} C_{i i} l\right) \mu_{i}\right] \cos \phi, \\
& \sum_{j=1}^{N} \sum_{n=1}^{K^{*}}\left[B_{j 1 n} B_{j i n}^{* *}\left(a_{i}, \mu_{i}, \phi\right)+C_{j 1 n} C_{j i n}^{* *}\left(a_{i}, \mu_{i}, \phi\right)+E_{j 1 n} E_{j i n}^{* *}\left(a_{i}, \mu_{i}, \phi\right)\right] \\
& =-\left(U_{i x}+a_{i} \Omega_{i y} \mu_{i}\right) \sin \phi \\
& -C_{\mathrm{si}} \frac{\eta E_{\infty}}{\rho \tilde{T}_{i}} \frac{1}{a_{i}}\left[\sum_{n=1}^{K} \bar{R}_{i 1 n}\left(a_{i}^{n}+n a_{i}^{n-1} k_{i}^{*} C_{t i} l\right)\left(1-\mu_{i}^{2}\right)^{1 / 2} P_{n}^{1}\left(\mu_{i}\right)+a_{i}+k_{i}^{*} C_{i i} l\right] \sin \phi
\end{aligned}
$$

where

$$
\begin{aligned}
& {\left[\begin{array}{l}
B_{j i n}^{*}\left(r_{i}, \mu_{i}, \phi_{i}\right) \\
C_{j i n}^{*}\left(r_{i}, \mu_{i}, \phi_{i}\right) \\
E_{j i n}^{*}\left(r_{i}, \mu_{i}, \phi_{i}\right)
\end{array}\right]=\left[\begin{array}{l}
B_{j i 1 n}^{(2)} \\
C_{j i 1 n}^{(2)} \\
E_{j i 1 n}^{(2)}
\end{array}\right]-\frac{C_{\mathrm{m} i} l}{r_{i}}\left\langle r_{i} \frac{\partial}{\partial r_{i}}\left[\begin{array}{l}
B_{j i 1 n}^{(2)} \\
C_{j i 1 n}^{(2)} \\
E_{j i 1 n}^{(2)}
\end{array}\right]-\left[\begin{array}{l}
B_{j i 1 n}^{(2)} \\
C_{j i 1 n}^{(2)} \\
E_{j i 1 n}^{(2)}
\end{array}\right]-\left(1-\mu_{i}^{2}\right)^{1 / 2} \frac{\partial}{\partial \mu_{i}}\left[\begin{array}{l}
B_{j 11 n}^{(1)} \\
C_{j i 1 n}^{(1)} \\
E_{j i 1 n}^{(1)}
\end{array}\right]\right),} \\
& {\left[\begin{array}{l}
B_{j i n}^{* *}\left(r_{i}, \mu_{i}, \phi_{i}\right) \\
C_{j i n}^{* *}\left(r_{i}, \mu_{i}, \phi_{i}\right) \\
E_{j i n}^{* *}\left(r_{i}, \mu_{i}, \phi_{i}\right)
\end{array}\right]=\left[\begin{array}{l}
B_{j i 1 n}^{(3)} \\
C_{j i 1 n}^{(3)} \\
E_{j i 1 n}^{(3)}
\end{array}\right]-\frac{C_{\mathrm{m} i} l}{r_{i}}\left\langle r_{i} \frac{\partial}{\partial r_{i}}\left[\begin{array}{l}
B_{j i 1 n}^{(3)} \\
C_{j i 1 n}^{(3)} \\
E_{j i 1 n}^{(3)}
\end{array}\right]-\left[\begin{array}{l}
B_{j i 1 n}^{(3)} \\
C_{j i 1 n}^{(3)} \\
E_{j i 1 n}^{(3)}
\end{array}\right]+\left(1-\mu_{i}^{2}\right)^{-1 / 2} \frac{\partial}{\partial \phi_{i}}\left[\begin{array}{l}
B_{j i 1 n}^{(1)} \\
C_{j i 1 n}^{(1)} \\
E_{j i 1 n}^{(1)}
\end{array}\right]\right)}
\end{aligned}
$$

Note that the dependence on $\phi$ in equation (16) will factor out for this special case. Application of equation (16) at $K^{*}$ discrete values of $\theta_{i}$ on the surface of each particle $i$ can generate a set of $3 N K^{*}$ linear algebraic equations for the $3 N K^{*}$ unknown coefficients $B_{j 1 n}$, $C_{j 1 n}$ and $E_{j 1 n}$. 


\subsection{Velocities of free spheres}

The force exerted by the fluid on the $i$ th sphere and the hydrodynamic torque experienced by the sphere about its center are given by (Happel and Brenner, 1983)

$$
\begin{gathered}
F_{i}=-4 \pi \nabla\left[r_{i}^{3} p_{-2}^{(i)}\right], \\
\underline{T}_{i}=-8 \pi \eta \nabla\left[r_{i}^{3} \chi_{-2}^{(i)}\right],
\end{gathered}
$$

which shows that only the lowest-order solid spherical harmonic functions contribute to the force and torque on each particle. Substitution of equation (13) into equation (19) leads to

$$
\begin{gathered}
{\underset{\sim}{i}}_{i}=-4 \pi\left(E_{i 11}{\underset{e}{e}}_{x}+F_{i 11}{\underset{\sim}{e}}_{y}+E_{i 01}{\underset{e}{z}}_{z}\right), \\
{\underset{\sim}{T}}_{i}=-8 \pi \eta\left(A_{i 11}{\underset{e}{x}}_{x}+B_{i 11}{\underset{\sim}{y}}_{y}+A_{i 01}{\underset{\sim}{z}}_{z}\right),
\end{gathered}
$$

where the six coefficients for each of the $N$ spheres are known from the collocation solution.

Because the particles are freely suspended in the surrounding fluid, the net force and torque exerted by the fluid on each sphere must vanish. Applying this constraint to equation (20), one has

$$
A_{i 01}=A_{i 11}=B_{i 11}=E_{i 01}=E_{i 11}=F_{i 11}=0, \quad i=1,2, \ldots, N .
$$

To determine the instantaneous translational and angular velocities $U_{i}$ and $\Omega_{i}$ of the $N$ particles ( $6 N$ components in total), the above $6 N$ equations must be solved simultaneously. The result can be expressed as

$$
\begin{gathered}
\underline{U}_{i}=\sum_{j=1}^{N} \underset{\approx}{M_{i j}} \cdot{\underset{\sim}{U}}_{j}^{(0)}, \\
a_{i} \Omega_{i}=\sum_{j=1}^{N} \underset{\approx}{N_{i j}} \cdot{\underset{\sim}{j}}_{j}^{(0)}, \quad i=1,2, \ldots, N,
\end{gathered}
$$

with

$$
\underline{\sim}_{j}^{(0)}=-A_{j} \nabla T_{\infty},
$$

which is the thermophoretic velocity of sphere $j$ in the absence of all the other ones. $A_{j}$ is used to represent the value of $A$ defined by equation (1b) for the undisturbed ther-

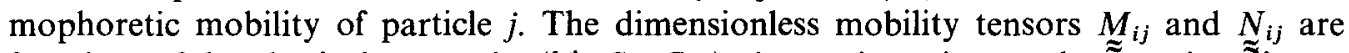
functions of the physical properties $\left(k_{i}^{*}, C_{t i}, C_{\mathrm{m} i}\right)$, sizes, orientations and separation distances of the particles. It can be shown that ${\underset{\tilde{E}}{i j}}_{i j}$ and ${\underset{\tilde{e}}{i j}}_{i j}$ are independent of the thermal slip coefficients $\left(C_{s i}\right)$ of the particles. When the $i$ th sphere is separated by an infinite distance from all of the others, it is evident that

$$
\begin{aligned}
& \underset{\approx}{M_{i i}}=\underset{\approx}{I}, \\
& \underset{\approx}{M_{i j}}=\underset{\approx}{0} \quad(j=1,2, \ldots, N \text { but } j \neq i), \\
& \stackrel{N_{i j}}{\approx} \underset{\approx}{0} \quad(j=1,2, \ldots, N),
\end{aligned}
$$

for $i=1,2, \ldots$, or $N$.

\subsection{Velocity of a rigid cluster of spheres}

We now consider the thermophoretic motion of a rigid cluster of $N$ spheres connected through their centers with rigid rods of arbitrary lengths. The connecting rods are assumed to be infinitesimally thin compared to the sphere sizes; hence they make neither thermal nor hydrodynamic contributions but only serve to ensure the rigid-body motion of the cluster. Here, our aim is to explore the thermophoresis of aggregates formed by flocculation of aerosol particles. 
For such a rigid cluster of spheres undergoing thermophoresis, all of the equations in the previous subsections except for equations (21) and (24) still apply. Since the angular velocity of a rigid body is independent of the choice of origin, one has

$$
\Omega_{i}=\Omega, \quad i=1,2, \ldots, N,
$$

where $\Omega$ is the angular velocity of the cluster. The translational velocity of each sphere in the rigid cluster can be written as

$$
\underline{\sim}_{i}={\underset{\sim}{U}}_{0}+\underset{\sim}{\Omega} \times{\underset{\sim}{0 i}}_{0 i}, \quad i=1,2, \ldots, N,
$$

where $\underline{U}_{0}$ refers to the translational velocity of a point on the cluster designated as the origin and $\underline{r}_{0 i}$ is the position vector of the center of sphere $i$ measured from the origin. Using equations (25) and (26) in Section 2.2, the set of unknown constants $\left\{A_{j m n}, B_{j m n}, \ldots, F_{j m n}\right\}$ or $\left\{B_{j 1 n}, C_{j 1 n}, E_{j 1 n}\right\}$ can be solved in terms of the components of the cluster velocities ${\underset{\sim}{0}}_{0}$ and $\Omega$ by the same collocation method.

To determine $U_{0}$ and $\Omega$ the constraint that the net force and net torque exerted by the fluid on the rigid cluster are zero is needed. Since the force acting on a rigid body is independent of the choice of origin, the net force on the cluster can be expressed in terms of the forces exerted on the individual spheres as

$$
\underline{\sim}=\sum_{i=1}^{N} \underline{F}_{i}=\underset{\sim}{0}
$$

The net torque about the cluster's origin can also be expressed in terms of the forces and torques acting on the $N$ spheres as

$$
\underline{\sim}_{0}=\sum_{i=1}^{N}\left(\underline{T}_{i}+\underline{\sim}_{0 i} \times{\underset{\sim}{F}}_{i}\right)=\underset{\sim}{0}
$$

Substitution of equation (20) into equations (27) and (28) leads to

$$
\begin{gathered}
\sum_{i=1}^{N} E_{i 11}=0, \\
\sum_{i=1}^{N} F_{i 11}=0 \\
\sum_{i=1}^{N} E_{i 01}=0, \\
\sum_{i=1}^{N}\left(2 \eta A_{i 11}+y_{0 i} E_{i 01}-z_{0 i} F_{i 11}\right)=0 \\
\sum_{i=1}^{N}\left(2 \eta B_{i 11}+z_{0 i} E_{i 11}-x_{0 i} E_{i 01}\right)=0 \\
\sum_{i=1}^{N}\left(2 \eta A_{i 01}+x_{0 i} F_{i 11}-y_{0 i} E_{i 11}\right)=0,
\end{gathered}
$$

where $x_{0 i}, y_{0 i}$ and $z_{0 i}$ are the components of vector $\underline{r}_{0 i}$ in Cartesian coordinates. The translational and angular velocities $\underline{U}_{0}$ and $\Omega$ (each having three components) can be determined by solving the above six equations simultaneously.

\section{RESULTS FOR TWO FREE SPHERES}

In this section we consider the thermophoretic motion of two freely suspended spheres which are oriented arbitrarily relative to the direction of the prescribed temperature 
Table 1. The mobility parameters for the thermophoresis of two identical spheres normal to the line of their centers with $C_{\mathrm{t}} l / a=0.2$ and $C_{\mathrm{m}} l / a=0.1$ (the values in parentheses are calculated from the approximate formulas obtained by the method of reflections for comparison)

\begin{tabular}{|c|c|c|c|c|c|c|c|}
\hline$k^{*}$ & $2 a / r_{12}$ & $M_{11}^{(n)}$ & $-M_{12}^{(n)}$ & $M_{11}^{(n)}+M_{12}^{(n)}$ & $N_{1 \text { I }}$ & $N_{12}$ & $N_{11}+N_{12}$ \\
\hline \multirow[t]{8}{*}{1} & 0.2 & 1.0000 & 0.0005 & $0.9996(0.9996)$ & $3.1 \mathrm{E}-7$ & $-3.4 \mathrm{E}-8$ & $2.7 \mathrm{E}-7(2.7 \mathrm{E}-7)$ \\
\hline & 0.4 & 1.0005 & 0.0040 & $0.9965(0.9965)$ & $4.3 \mathrm{E}-5$ & $-4.2 \mathrm{E}-6$ & $3.9 \mathrm{E}-5(3.4 \mathrm{E}-5)$ \\
\hline & 0.6 & 1.0017 & 0.0135 & $0.9881(0.9881)$ & $8.8 \mathrm{E}-4$ & $-4.2 \mathrm{E}-5$ & $8.4 \mathrm{E}-4(6.4 \mathrm{E}-4)$ \\
\hline & 0.8 & 1.0054 & 0.0325 & $0.9729(0.9717)$ & $8.8 \mathrm{E}-3$ & $6.2 E-4$ & $9.4 \mathrm{E}-3(4.4 \mathrm{E}-3)$ \\
\hline & 0.9 & 1.0112 & 0.0468 & $0.9643(0.9596)$ & 0.0260 & 0.0048 & $0.0307(9.9 \mathrm{E}-3)$ \\
\hline & 0.95 & 1.0175 & 0.0553 & 0.9623 & 0.0468 & 0.0128 & 0.0596 \\
\hline & 0.99 & 1.0280 & 0.0612 & 0.9667 & 0.0849 & 0.0345 & 0.1194 \\
\hline & 1.0 & 1.128 & -0.034 & 1.161 & 0.565 & 0.509 & 1.075 \\
\hline \multirow[t]{8}{*}{10} & 0.2 & 0.9996 & 0.0005 & $0.9991(0.9991)$ & $3.1 \mathrm{E}-7$ & $3.1 \mathrm{E}-7$ & $6.2 \mathrm{E}-7(6.0 \mathrm{E}-7)$ \\
\hline & 0.4 & 0.9965 & 0.0040 & $0.9925(0.9925)$ & $4.4 \mathrm{E}-5$ & $4.5 E-5$ & $8.9 \mathrm{E}-5(7.7 \mathrm{E}-5)$ \\
\hline & 0.6 & 0.9884 & 0.0132 & $0.9753(0.9750)$ & $9.3 \mathrm{E}-4$ & $9.6 \mathrm{E}-4$ & $1.9 \mathrm{E}-3(1.3 \mathrm{E}-3)$ \\
\hline & 0.8 & 0.9744 & 0.0287 & $0.9457(0.9417)$ & 0.0099 & 0.0106 & $0.0205(9.8 \mathrm{E}-3)$ \\
\hline & 0.9 & 0.9671 & 0.0356 & $0.9315(0.9180)$ & 0.0297 & 0.0330 & $0.0627(0.0224)$ \\
\hline & 0.95 & 0.9650 & 0.0357 & 0.9293 & 0.0528 & 0.0600 & 0.1127 \\
\hline & 0.99 & 0.9662 & 0.0305 & 0.9357 & 0.0897 & 0.1040 & 0.1937 \\
\hline & 1.0 & 0.985 & 0.010 & 0.975 & 0.190 & 0.207 & 0.398 \\
\hline \multirow[t]{8}{*}{100} & 0.2 & 0.9994 & 0.0005 & $0.9989(0.9989)$ & $3.1 \mathrm{E}-7$ & $4.4 \mathrm{E}-7$ & $7.5 \mathrm{E}-7(7.3 \mathrm{E}-7)$ \\
\hline & 0.4 & 0.9956 & 0.0040 & $0.9916(0.9916)$ & $4.4 \mathrm{E}-5$ & $6.4 \mathrm{E}-5$ & 1.1E-4 (9.3E-5) \\
\hline & 0.6 & 0.9853 & 0.0131 & $0.9722(0.9719)$ & $9.5 \mathrm{E}-4$ & $1.4 \mathrm{E}-3$ & $2.3 \mathrm{E}-3(1.6 \mathrm{E}-3)$ \\
\hline & 0.8 & 0.9674 & 0.0275 & $0.9399(0.9348)$ & 0.0104 & 0.0147 & $0.0251(0.0120)$ \\
\hline & 0.9 & 0.9573 & 0.0317 & $0.9256(0.9086)$ & 0.0315 & 0.0444 & $0.0759(0.0273)$ \\
\hline & 0.95 & 0.9534 & 0.0288 & 0.9246 & 0.0558 & 0.0788 & 0.1346 \\
\hline & 0.99 & 0.9524 & 0.0195 & 0.9329 & 0.0927 & 0.1313 & 0.2240 \\
\hline & 1.0 & 0.944 & 0.025 & 0.919 & 0.063 & 0.107 & 0.170 \\
\hline \multirow[t]{8}{*}{1000} & 0.2 & 0.9994 & 0.0005 & $0.9989(0.9989)$ & $3.1 \mathrm{E}-7$ & $4.6 \mathrm{E}-7$ & 7.7E-7 (7.5E-7) \\
\hline & 0.4 & 0.9955 & 0.0040 & $0.9915(0.9915)$ & $4.4 \mathrm{E}-5$ & $6.7 \mathrm{E}-5$ & $1.1 \mathrm{E}-4(9.6 \mathrm{E}-5)$ \\
\hline & 0.6 & 0.9850 & 0.0130 & $0.9719(0.9716)$ & $9.5 \mathrm{E}-4$ & $1.4 \mathrm{E}-3$ & $2.4 \mathrm{E}-3(1.6 \mathrm{E}-3)$ \\
\hline & 0.8 & 0.9666 & 0.0273 & $0.9393(0.9340)$ & 0.0105 & 0.0153 & $0.0257(0.0123)$ \\
\hline & 0.9 & 0.9562 & 0.0311 & $0.9250(0.9076)$ & 0.0318 & 0.0460 & $0.0778(0.0279)$ \\
\hline & 0.95 & 0.9521 & 0.0278 & 0.9243 & 0.0563 & 0.0815 & 0.1378 \\
\hline & 0.99 & 0.9579 & 0.0180 & 0.9328 & 0.0932 & 0.1351 & 0.2283 \\
\hline & 1.0 & 0.938 & 0.027 & 0.911 & 0.045 & 0.093 & 0.138 \\
\hline
\end{tabular}

gradient. For this simple case, equations (22a) and (22b) for the translational and rotational velocities of the particles become

$$
\begin{gathered}
\underline{\sim}_{i}=\sum_{j=1}^{N}\left[M_{i j}^{(p)} \underset{\sim}{e}+M_{i j}^{(n)}(\underset{\sim}{I}-\underset{\sim}{e})\right] \cdot \underline{\sim}_{j}^{(0)}, \\
a_{i} \underline{\Omega}_{i}=-\sum_{j=1}^{N} N_{i j} \underset{\sim}{e} \times{\underset{\sim}{j}}_{j}^{(0)},
\end{gathered}
$$

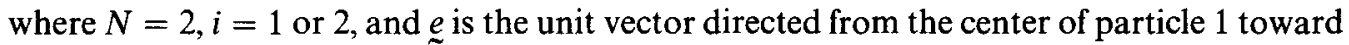
the center of particle 2 . Using a method of reflections, the formulas for the mobility parameters $M_{i j}^{(p)}, M_{i j}^{(n)}$ and $N_{i j}$ were derived in power series of $r_{12}^{-1}$ up to $\mathrm{O}\left(r_{12}^{-7}\right)$, where $r_{12}$ is the center-to-center distance between the two spheres (Keh and Chen, 1995).

On the other hand, the exact solution of the mobility parameters $M_{11}^{(p)}, M_{12}^{(p)}, M_{21}^{(p)}$ and $M_{22}^{(p)}$ for the thermophoresis of two arbitrary spheres along the line of their centers was presented by utilizing spherical bipolar coordinates (Chen and Keh, 1996). The combined analytical-numerical solution of these particle interaction parameters, resulting from using the boundary collocation technique for the axisymmetric motion of multiple spheres, was also obtained (Chen and Keh, 1996). It was found that the collocation results agree very well with the exact solution for various sizes, spacings and physical properties of the two particles. In this section the numerical results of the remaining eight parameters $\left(M_{11}^{(n)}, M_{12}^{(n)}\right.$, $M_{21}^{(n)}, M_{22}^{(n)}, N_{11}, N_{12}, N_{21}$, and $N_{22}$ ), obtained by using the boundary-collocation, truncated-series method described in the previous section, will be presented. The accuracy 
Table 2. The mobility parameters $M_{i j}^{(n)}$ and $N_{i j}$ for the thermophoresis of two spheres of unequal radii with the same physical properties $\left(k^{*}=100, C_{1} l / a_{1}=0.2\right.$ and $\left.C_{\mathrm{m}} l / a_{1}=0.1\right)$

\begin{tabular}{llllllllll}
\hline$\frac{a_{2}}{a_{1}}$ & $\frac{a_{1}+a_{2}}{r_{12}}$ & $M_{11}^{(n)}$ & $-M_{12}^{(n)}$ & $-M_{21}^{(n)}$ & $M_{22}^{(n)}$ & $N_{11}$ & $N_{12}$ & $-N_{21}$ & $-N_{22}$ \\
\hline 0.5 & 0.2 & 0.9999 & 0.0001 & 0.0012 & 0.9987 & $2.5 \mathrm{E}-7$ & $3.4 \mathrm{E}-7$ & $1.3 \mathrm{E}-7$ & $1.6 \mathrm{E}-7$ \\
& 0.4 & 0.9992 & 0.0012 & 0.0095 & 0.9894 & $3.3 \mathrm{E}-5$ & $4.5 \mathrm{E}-5$ & $2.0 \mathrm{E}-5$ & $2.3 \mathrm{E}-5$ \\
& 0.6 & 0.9976 & 0.0036 & 0.0319 & 0.9644 & 0.0006 & 0.0008 & 0.0005 & 0.0006 \\
& 0.8 & 0.9960 & 0.0062 & 0.0754 & 0.9150 & 0.0055 & 0.0076 & 0.0077 & 0.0079 \\
& 0.9 & 0.9972 & 0.0042 & 0.1069 & 0.8762 & 0.0152 & 0.0211 & 0.0285 & 0.0274 \\
& 0.95 & 1.0001 & -0.0002 & 0.1249 & 0.8506 & 0.0261 & 0.0362 & 0.0576 & 0.0531 \\
& 0.99 & 1.0064 & -0.0104 & 0.1423 & 0.8197 & 0.0458 & 0.0648 & 0.1145 & 0.1067 \\
& 0.995 & 1.01 & -0.01 & 0.14 & 0.82 & 0.05 & 0.06 & 0.12 & 0.11 \\
2 & 0.2 & 0.9983 & 0.0009 & 0.0001 & 0.7856 & $1.4 \mathrm{E}-7$ & $1.5 \mathrm{E}-7$ & $5.4 \mathrm{E}-7$ & $2.2 \mathrm{E}-7$ \\
& 0.4 & 0.9862 & 0.0074 & 0.0012 & 0.7847 & $2.1 \mathrm{E}-5$ & $2.4 \mathrm{E}-5$ & $7.2 \mathrm{E}-5$ & $3.0 \mathrm{E}-5$ \\
& 0.6 & 0.9535 & 0.0250 & 0.0035 & 0.7825 & 0.0005 & 0.0006 & 0.0014 & 0.0006 \\
& 0.8 & 0.8902 & 0.0582 & 0.0052 & 0.7797 & 0.0076 & 0.0082 & 0.0119 & 0.0051 \\
& 0.9 & 0.8433 & 0.0800 & 0.0017 & 0.7797 & 0.0275 & 0.0287 & 0.0314 & 0.0139 \\
& 0.95 & 0.8161 & 0.0896 & -0.0035 & 0.7810 & 0.0550 & 0.0555 & 0.0513 & 0.0230 \\
& 0.99 & 0.7955 & 0.0926 & -0.0112 & 0.7832 & 0.1048 & 0.0997 & 0.0780 & 0.0351 \\
1.0 & 0.73 & -0.08 & -0.05 & 0.90 & 0.17 & 0.00 & 0.14 & -0.17 \\
5 & 0.2 & 0.9960 & 0.0017 & $1.8 \mathrm{E}-5$ & 0.7428 & $1.4 \mathrm{E}-7$ & $1.8 \mathrm{E}-8$ & $1.9 \mathrm{E}-7$ & $6.5 \mathrm{E}-8$ \\
& 0.4 & 0.9680 & 0.0137 & 0.0001 & 0.7427 & $3.1 \mathrm{E}-6$ & $3.2 \mathrm{E}-6$ & $2.2 \mathrm{E}-5$ & $8.3 \mathrm{E}-6$ \\
& 0.6 & 0.8921 & 0.0464 & 0.0003 & 0.7425 & 0.0001 & 0.0001 & 0.0004 & 0.0001 \\
& 0.8 & 0.7431 & 0.1105 & -0.0002 & 0.7425 & 0.0022 & 0.0022 & 0.0034 & 0.0012 \\
0.9 & 0.6299 & 0.1584 & -0.0020 & 0.7430 & 0.0118 & 0.0112 & 0.0082 & 0.0029 \\
0.95 & 0.5602 & 0.1860 & -0.0038 & 0.7437 & 0.0308 & 0.0280 & 0.0129 & 0.0047 \\
0.99 & 0.5013 & 0.2051 & -0.0065 & 0.7446 & 0.0806 & 0.0658 & 0.0191 & 0.0071 \\
\hline
\end{tabular}

of this solution technique will be tested by comparing the results with the asymptotic solution given in Keh and Chen (1995).

The details of the collocation scheme used for this work are given in Keh and Yang (1991) and a DEC 3000/600 AXP workstation was utilized to perform the calculations. All of the numerical results presented in Tables 1-5 converge to at least the digits as shown with reasonable choices of $K$ and $K^{*}$. A number of collocation solutions of the interaction parameters $M_{11}^{(n)}, M_{12}^{(n)}, N_{11}$ and $N_{12}$ for the thermophoretic motion of two identical spheres $\left(a_{1}=a_{2}=a, C_{\mathrm{t} 1}=C_{\mathrm{t} 2}=C_{\mathrm{t}}, C_{\mathrm{m} 1}=C_{\mathrm{m} 2}=C_{\mathrm{m}}, k_{1}^{*}=k_{2}^{*}=k^{*}\right.$ and $\left.A_{1}=A_{2}\right)$ with various relative thermal conductivities and spacings are presented in Table 1 . The two identical spheres will translate at the same velocity (because $M_{21}^{(n)}=M_{12}^{(n)}, M_{22}^{(n)}=M_{11}^{(n)}$ and $\left.U_{2}^{(0)}=U_{1}^{(0)}\right)$ and rotate with angular velocities equal in magnitude but opposite in direction (since $N_{22}=-N_{11}$ and $N_{21}=-N_{12}$ ). Note that the spheres are still allowed to rotate freely when they are touched with each other $\left(2 a / r_{12}=1\right)$. The asymptotic solutions for $M_{11}^{(n)}+M_{12}^{(n)}$ and $N_{11}+N_{12}$ accurate to $\mathrm{O}\left(r_{12}^{-7}\right)$ obtained by using the method of reflections are also listed (in the parentheses) in Table 1 for comparison. It can be seen that these method-of-reflections results agree quite well with the collocation results so long as the particle surfaces are more than $\frac{2}{3}$ of the sum of the radii apart (i.e. $2 a / r_{12} \leqslant 0.6$ ). However, the accuracy of the asymptotic solutions (especially for $N_{i j}$ ) deteriorates rapidly, as expected, when the particles get close to each other. While the reflection results accurate to $\mathrm{O}\left(r_{12}^{-7}\right)$ always underestimate the value of $M_{11}^{(p)}+M_{12}^{(p)}$ (Keh and Chen, 1995), they also underestimate the values of $M_{11}^{(n)}+M_{12}^{(n)}$ and $N_{11}+N_{12}$. Note that, the direction of rotation (relative to the direction of translation) of two spheres undergoing thermophoresis is opposite to that of two settling spheres.

Some numerical values of the mobility parameters $M_{i j}^{(n)}$ and $N_{i j}$ for the thermophoretic motion of two different-sized spheres with the same physical properties $C_{\mathrm{t} 1}=C_{\mathrm{t} 2}=C_{\mathrm{t}}$, $C_{\mathrm{m} 1}=C_{\mathrm{m} 2}=C_{\mathrm{m}}$ and $k_{1}^{*}=k_{2}^{*}=k^{*}$ ) are listed in Table 2. Also, in Fig. 2, the normalized translational and rotational velocities of particle 1 of two spheres which have the same physical properties and experience thermophoresis perpendicular to the line through their centers are plotted as a function of $\left(a_{1}+a_{2}\right) / r_{12}$ with $a_{2} / a_{1}$ as a parameter. The results in 

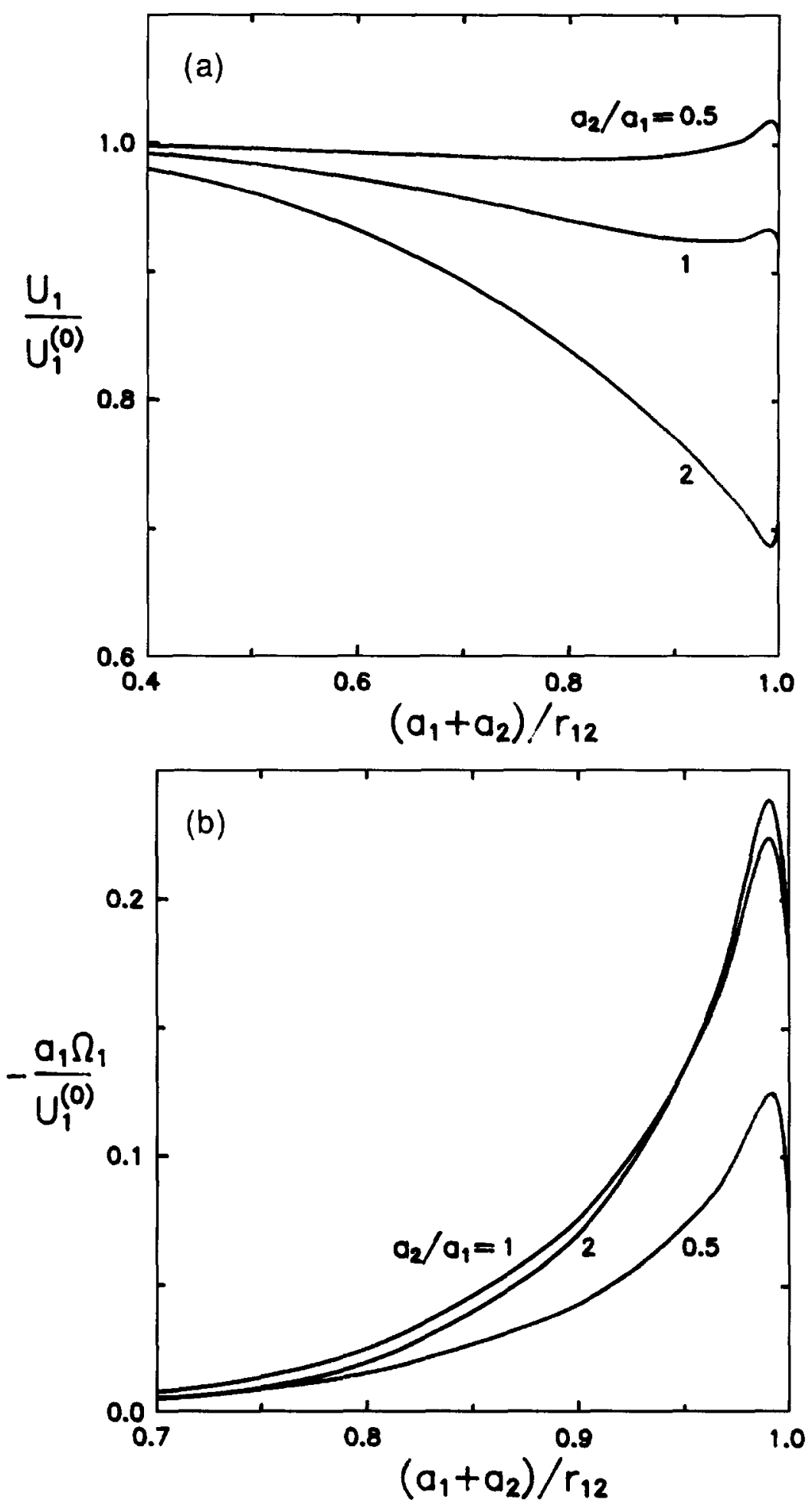

Fig. 2. Plots of the normalized translational and rotational velocities of two spheres of identical thermal conductivities and surface properties with $k^{*}=100, C_{\mathrm{t}} l / a_{1}=0.2$ and $C_{\mathrm{m}} l / a_{1}=0.1$ undergoing thermophoresis perpendicular to the line of their centers versus the separation parameter $\left(a_{1}+a_{2}\right) / r_{12}$ with $a_{2} / a_{1}$ as a parameter. 
Tables 1 and 2 and Fig. 2 illustrate that the particles' interaction decreases rapidly, for all values of $k^{*}$ and $a_{2} / a_{1}$, with an increase in the gap between them (i.e. decreasing $\left.\left(a_{1}+a_{2}\right) / r_{12}\right)$. However, the interaction between particles can be strong when the surface-to-surface spacing approaches zero. The effect of the interaction, in general, is greater on the smaller of the spheres than on the larger one for given values of $k^{*}$ and $\left(a_{1}+a_{2}\right) / r_{12}$. Note that the translational and rotational velocities of the particles are not necessarily a monotonic increasing functions of the separation parameter $\left(a_{1}+a_{2}\right) / r_{12}$. These complex results are generated from the combined effects of particle interactions on local temperature and fluid velocity fields.

\section{RESULTS FOR THREE COAXIAL FREE SPHERES}

In the previous section solutions for the thermophoresis of two spherical particles based on the collocation technique have been presented and were shown to be in good agreement with the method-of-reflection results. This section will examine the solutions for the thermophoretic motion of three spheres using the same collocation method. Since the number of particle interaction parameters in the general problem of three spheres is so great, here we only consider the motion of three coaxial spheres with the same physical properties in a symmetric configuration that the two end particles have the same radius and distance from the central one $\left(a_{1}=a_{3}\right.$ and $\left.r_{12}=r_{23}\right)$. For this linear and symmetric case, equation (31) for the translational and rotational velocities is still valid (now with $N=3$ and $i=1,2$ or 3 ) and one has ${\underset{\sim}{1}}_{1}=\underline{U}_{3}, \underline{\Omega}_{1}=-\underline{\Omega}_{3}$ and $\underline{\Omega}_{2}=\underline{0}$, or

$$
\begin{gathered}
M_{11}^{(p, n)}=M_{33}^{(p, n)}, \\
M_{12}^{(p, n)}=M_{32}^{(p, n)}, \\
M_{23}^{(p, n)}=M_{21}^{(p, n)}, \\
M_{31}^{(p, n)}=M_{13}^{(p, n)}, \\
N_{11}=-N_{33}, \\
N_{12}=-N_{32}, \\
N_{23}=-N_{21}, \\
N_{31}=-N_{13}, \\
N_{22}=0 .
\end{gathered}
$$

One may wonder what the shielding effect of neighboring particles on thermophoresis is. For the particle-interaction effects on the motion of a sphere (say, sphere 1) in an aerosol, the contribution of the closest neighbors should be carried in terms of parameters $M_{12}^{(p, n)}$ and the contribution of the next layer of neighbors is conducted through parameters $M_{13}^{(p, n)}$ for the above-mentioned linear and symmetric case. A comparison between the magnitudes of $M_{12}^{(p, n)}$ and $M_{13}^{(p, n)}$ gives the shielding effect. The numerical solution of $M_{i j}^{(p)}$ for this case, resulting from using the collocation method for the axisymmetric motion of three spheres, was obtained by Chen and Keh (1996). These collocation results compare quite favorably with the formulas analytically derived.

In Table 3, numerical results of the mobility parameters $M_{i j}^{(n)}$ and $N_{i j}$ for the thermophoretic motion of three coaxial spheres are presented for three cases of relative radii. The normalized translational and rotational velocities of these particles undergoing thermophoresis perpendicular to the line through their centers are also listed in the same table. In general, the particle interactions decrease with increasing gap thickness between two neighboring particles. However, similar to the case of two particles, the mobility parameters or normalized particle velocities are not necessarily a monotonic function of the separation 
parameter $\left(a_{1}+a_{2}\right) / r_{12}$. The collocation results for the more general case of three coaxial spheres, such as $a_{1} \neq a_{3}, r_{12} \neq r_{23}$ or the situation that the physical properties of the particles are different, can be obtained without any further difficulty. However, all 27 mobility parameters, instead of 14 , are required to compute the particle velocities.

For the thermophoretic motion of three identical spheres along the line of their centers, it was found that the presence of an end sphere is to enhance the two-particle interaction effect on the other two spheres (Chen and Keh, 1996). One may be interested to see how significantly the existence of a third collinear sphere affects the thermophoretic velocities of

Table 3. The mobility parameter $M_{i j}^{(n)}$ and $N_{i j}$ for the thermophoresis of three coaxial spheres with the same physical properties $\left(k^{*}=100, C_{1} l / a_{1}=0.2\right.$ and $\left.C_{m} l / a_{1}=0.1\right)$ for the symmetric case of $a_{1}=a_{3}$ and $r_{12}=r_{23}$

\begin{tabular}{|c|c|c|c|c|c|c|c|c|}
\hline$a_{1}: a_{2}: a_{3}$ & $\frac{a_{1}+a_{2}}{r_{12}}$ & $-M_{12}^{(n)}$ & $-M_{23}^{(n)}$ & $-M_{31}^{(n)}$ & $M_{11}^{(n)}$ & $M_{22}^{(n)}$ & $U_{1} / U_{i}^{(0)}$ & $U_{2} / U_{2}^{(0)}$ \\
\hline \multirow[t]{8}{*}{$1: 1: 1$} & 0.2 & 0.0005 & 0.0005 & 0.0001 & 0.9994 & 0.9989 & 0.9988 & 0.9979 \\
\hline & 0.4 & 0.0040 & 0.0040 & 0.0005 & 0.9950 & 0.9912 & 0.9906 & 0.9832 \\
\hline & 0.6 & 0.0133 & 0.0130 & 0.0019 & 0.9837 & 0.9707 & 0.9685 & 0.9447 \\
\hline & 0.8 & 0.0301 & 0.0270 & 0.0064 & 0.9640 & 0.9352 & 0.9276 & 0.8811 \\
\hline & 0.9 & 0.0391 & 0.0304 & 0.0126 & 0.9528 & 0.9160 & 0.9011 & 0.8552 \\
\hline & 0.95 & 0.0416 & 0.0262 & 0.0186 & 0.9482 & 0.9099 & 0.8879 & 0.8576 \\
\hline & 0.99 & 0.0404 & 0.0143 & 0.0268 & 0.9461 & 0.9116 & 0.8788 & 0.8831 \\
\hline & 1.0 & 0.0442 & 0.0090 & 0.0295 & 0.9434 & 0.9118 & 0.8697 & 0.8937 \\
\hline \multirow[t]{7}{*}{$1: 2: 1$} & 0.2 & 0.0018 & 0.0001 & 0.0000 & 0.9982 & 0.9997 & 0.9974 & 0.9992 \\
\hline & 0.4 & 0.0095 & 0.0012 & 0.0002 & 0.9860 & 0.9974 & 0.9796 & 0.9939 \\
\hline & 0.6 & 0.0321 & 0.0035 & 0.0007 & 0.9531 & 0.9919 & 0.9310 & 0.9814 \\
\hline & 0.8 & 0.0769 & 0.0041 & 0.0037 & 0.8887 & 0.9859 & 0.8339 & 0.9734 \\
\hline & 0.9 & 0.1095 & -0.0020 & 0.0088 & 0.8383 & 0.9896 & 0.7566 & 0.9957 \\
\hline & 0.95 & 0.1274 & -0.0112 & 0.0145 & 0.8040 & 0.9985 & 0.7047 & 1.0320 \\
\hline & 0.99 & 0.14 & -0.03 & 0.02 & 0.77 & 1.01 & 0.65 & 1.09 \\
\hline \multirow[t]{8}{*}{$2: 1: 2$} & 0.2 & 0.0001 & 0.0012 & 0.0001 & 0.9997 & 0.9974 & 0.9994 & 0.9955 \\
\hline & 0.4 & 0.0012 & 0.0094 & 0.0012 & 0.9979 & 0.9789 & 0.9952 & 0.9644 \\
\hline & 0.6 & 0.0037 & 0.0316 & 0.0041 & 0.9933 & 0.9293 & 0.9843 & 0.8808 \\
\hline & 0.8 & 0.0077 & 0.0726 & 0.0108 & 0.9861 & 0.8332 & 0.9653 & 0.7219 \\
\hline & 0.9 & 0.0087 & 0.0992 & 0.0172 & 0.9836 & 0.7611 & 0.9550 & 0.6092 \\
\hline & 0.95 & 0.0080 & 0.1121 & 0.0224 & 0.9842 & 0.7159 & 0.9514 & 0.5442 \\
\hline & 0.99 & 0.0053 & 0.1202 & 0.0285 & 0.9876 & 0.6717 & 0.9522 & 0.4875 \\
\hline & 1.0 & -0.04 & -0.14 & -0.02 & 0.87 & 0.32 & 0.94 & 0.54 \\
\hline$a_{1}: a_{2}: a_{3}$ & $\frac{a_{1}+a_{2}}{r_{12}}$ & $N_{12}$ & $N_{23}$ & $N_{31}$ & $N_{11}$ & \multicolumn{2}{|c|}{$-a_{1} \Omega_{1} / U_{1}^{(0)}$} & \\
\hline \multirow[t]{8}{*}{$1: 1: 1$} & 0.2 & $6.9 \mathrm{E}-8$ & $4.6 \mathrm{E}-7$ & 2.3E-7 & $3.2 \mathrm{E}-7$ & \multicolumn{2}{|c|}{$1.5 \mathrm{E}-7$} & \\
\hline & 0.4 & $2.1 \mathrm{E}-5$ & $6.6 \mathrm{E}-5$ & $2.7 \mathrm{E}-5$ & $4.5 \mathrm{E}-5$ & \multicolumn{2}{|c|}{$3.9 \mathrm{E}-5$} & \\
\hline & 0.6 & $7.5 \mathrm{E}-4$ & $1.4 \mathrm{E}-3$ & $3.9 \mathrm{E}-4$ & $9.5 \mathrm{E}-4$ & \multicolumn{2}{|c|}{$1.3 \mathrm{E}-3$} & \\
\hline & 0.8 & 0.0109 & 0.0146 & 0.0024 & 0.0104 & \multicolumn{2}{|c|}{0.0189} & \\
\hline & 0.9 & 0.0366 & 0.0428 & 0.0047 & 0.0315 & \multicolumn{2}{|c|}{0.0634} & \\
\hline & 0.95 & 0.0681 & 0.0743 & 0.0058 & 0.0558 & \multicolumn{2}{|c|}{0.1180} & \\
\hline & 0.99 & 0.1185 & 0.1199 & 0.0049 & 0.0926 & \multicolumn{2}{|c|}{0.2063} & \\
\hline & 1.0 & 0.1179 & 0.0805 & -0.0019 & 0.0929 & \multicolumn{2}{|c|}{0.2128} & \\
\hline \multirow[t]{7}{*}{$1: 2: 1$} & 0.2 & $5.1 \mathrm{E}-8$ & $5.4 \mathrm{E}-7$ & $1.1 \mathrm{E}-7$ & $1.8 \mathrm{E}-7$ & \multirow{2}{*}{\multicolumn{2}{|c|}{$\begin{array}{l}9.6 \mathrm{E}-8 \\
2.7 \mathrm{E}-5\end{array}$}} & \\
\hline & 0.4 & $1.9 \mathrm{E}-5$ & $7.2 \mathrm{E}-5$ & $1.2 \mathrm{E}-5$ & $2.6 \mathrm{E}-5$ & \multirow{2}{*}{\multicolumn{2}{|c|}{$\begin{array}{l}2.7 \mathrm{E}-5 \\
1.0 \mathrm{E}-3\end{array}$}} & \\
\hline & 0.6 & $7.7 \mathrm{E}-4$ & $1.4 \mathrm{E}-3$ & $1.7 \mathrm{E}-4$ & $6.9 \mathrm{E}-4$ & & & \\
\hline & 0.8 & 0.0134 & 0.0120 & $8.4 \mathrm{E}-4$ & 0.0100 & \multicolumn{2}{|c|}{0.0181} & \\
\hline & 0.9 & 0.0509 & 0.0324 & $1.2 \mathrm{E}-3$ & 0.0349 & \multicolumn{2}{|c|}{0.0676} & \\
\hline & 0.95 & 0.1015 & 0.0540 & $6.0 \mathrm{E}-4$ & 0.0661 & \multicolumn{2}{|c|}{0.1331} & \\
\hline & 0.99 & 0.18 & 0.08 & 0.00 & 0.12 & \multicolumn{2}{|c|}{0.24} & \\
\hline $2: 1: 2$ & 0.2 & $4.6 \mathrm{E}-8$ & $1.6 \mathrm{E}-7$ & $1.9 \mathrm{E}-7$ & $2.7 \mathrm{E}-7$ & & E-7 & \\
\hline & 0.4 & $1.0 \mathrm{E}-5$ & $2.5 \mathrm{E}-5$ & $2.2 \mathrm{E}-5$ & $3.5 \mathrm{E}-5$ & & E-5 & \\
\hline & 0.6 & $2.9 \mathrm{E}-4$ & $6.3 \mathrm{E}-4$ & $3.3 \mathrm{E}-4$ & $6.6 \mathrm{E}-4$ & & E-4 & \\
\hline & 0.8 & 0.0038 & 0.0085 & 0.0021 & 0.0058 & & 086 & \\
\hline & 0.9 & 0.0119 & 0.0294 & 0.0046 & 0.0159 & & 269 & \\
\hline & 0.95 & 0.0220 & 0.0563 & 0.0068 & 0.0269 & & 489 & \\
\hline & 0.99 & 0.0395 & 0.1011 & 0.0090 & 0.0440 & & 864 & \\
\hline & 1.0 & 0.22 & 0.00 & -0.04 & -0.27 & & & \\
\hline
\end{tabular}


its two nearby particles when the temperature gradient is imposed normal to the line of their centers. In Fig. 3, the normalized translational and rotational velocities of three identical spheres with equal spacings undergoing thermophoresis perpendicular to the line of their centers are plotted by solid curves as a function of separation parameter $2 a / r_{12}$. The
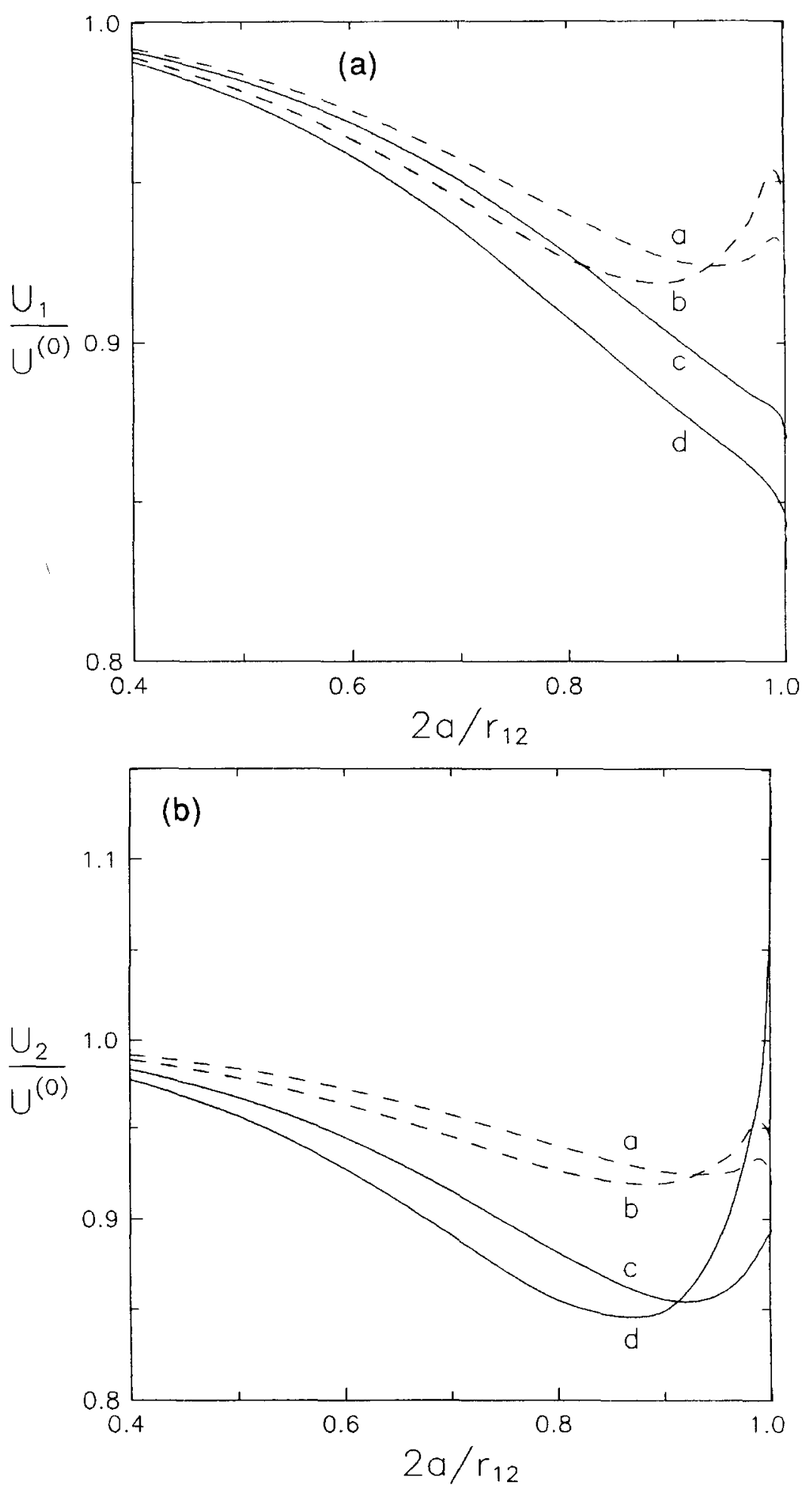

Fig. 3(a) and (b). 


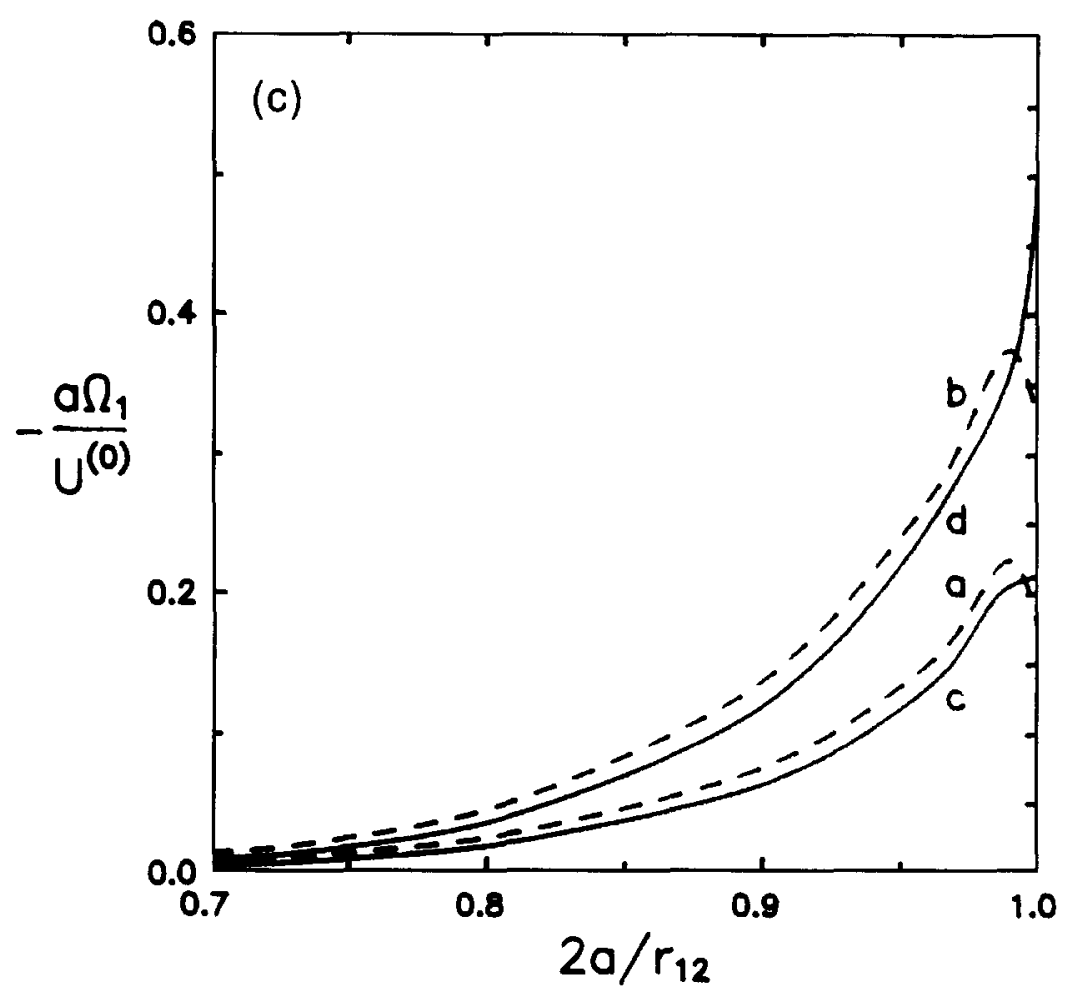

Fig. 3. Plots of the normalized translational and rotational velocities of three identical coaxial spheres of radius $a$ with equal spacings undergoing thermophoresis perpendicular to the line of their centers versus the separation parameter $2 a / r_{12}:$ (a) translational velocity of particle 1 (or particle 3 ); (b) translational velocity of particle 2; (c) rotational velocity of particle 1 (or particle 3). For comparison, the dashed curves are plotted for the particle velocities when only two spheres are present. For curves (a) and (c), $C_{1} l / a=0.2, C_{\mathrm{m}} l / a=0.1$ and $k^{*}=100$; for curves (b) and (d), $C_{\mathrm{t}} l / a=0.02, C_{\mathrm{m}} l / a=0.01$ and $k^{*}=100$.

corresponding values of the first and second spheres when the third one is not present are plotted by dashed curves in the same figure for comparison. Similar to the case of the motion of three spheres parallel to the line of their centers, the existence of the third sphere in general is to increase the two-particle interaction effect on the thermophoretic migration velocities of the other two spheres. On the other hand, the existence of the third sphere is to decrease the two-particle interaction effect on the rotational velocities of the other two spheres, with exceptions when the spacing between two neighboring spheres gets close to zero. For small to moderate values of $2 a / r_{12}$, the thermophoretic velocity of the central sphere is smaller than that of the end ones. However, when the particles are close together, the central sphere migrates faster than the end ones. On the contrary, the migration velocity of the central sphere is always smaller than that of the end ones for the thermophoresis of three identical spheres along the line through their centers (Chen and Keh, 1996). Note that the shielding effect of neighboring spheres on thermophoresis can also be observed from Fig. 3a.

The normalized translational and rotational velocities of three identical spheres with equal spacings undergoing thermophoresis perpendicular to the line of their centers are plotted versus the conductivity ratio $k^{*}$ in Fig. 4 with $C_{\mathrm{t}} l / a$ and $C_{\mathrm{m}} l / a$ as parameters for the case of $2 a / r_{12}=0.8$. It can be found that the particle interaction effect in general is more significant if the value of $k^{*}$ becomes greater or the values of $C_{\mathrm{t}} l / a$ and $C_{\mathrm{m}} l / a$ become smaller (with exceptions for the rotation of the end spheres). In the limit $k^{*}=0$ or in the limit $C_{1} l / a \rightarrow \infty$, our collocation results show that the thermophoretic velocity of each of these three identical spheres is unaffected by the presence of the others; i.e. each sphere translates in the same velocity as it is isolated with no rotation. 


\section{RESULTS FOR A RIGID CLUSTER OF TWO SPHERES}

The translational and angular velocities of a rigid cluster of spheres undergoing thermophoresis can also be determined by the procedure described in Section 2. For conciseness, here we only consider the motion of a dumbbell, the cluster composed of two spheres
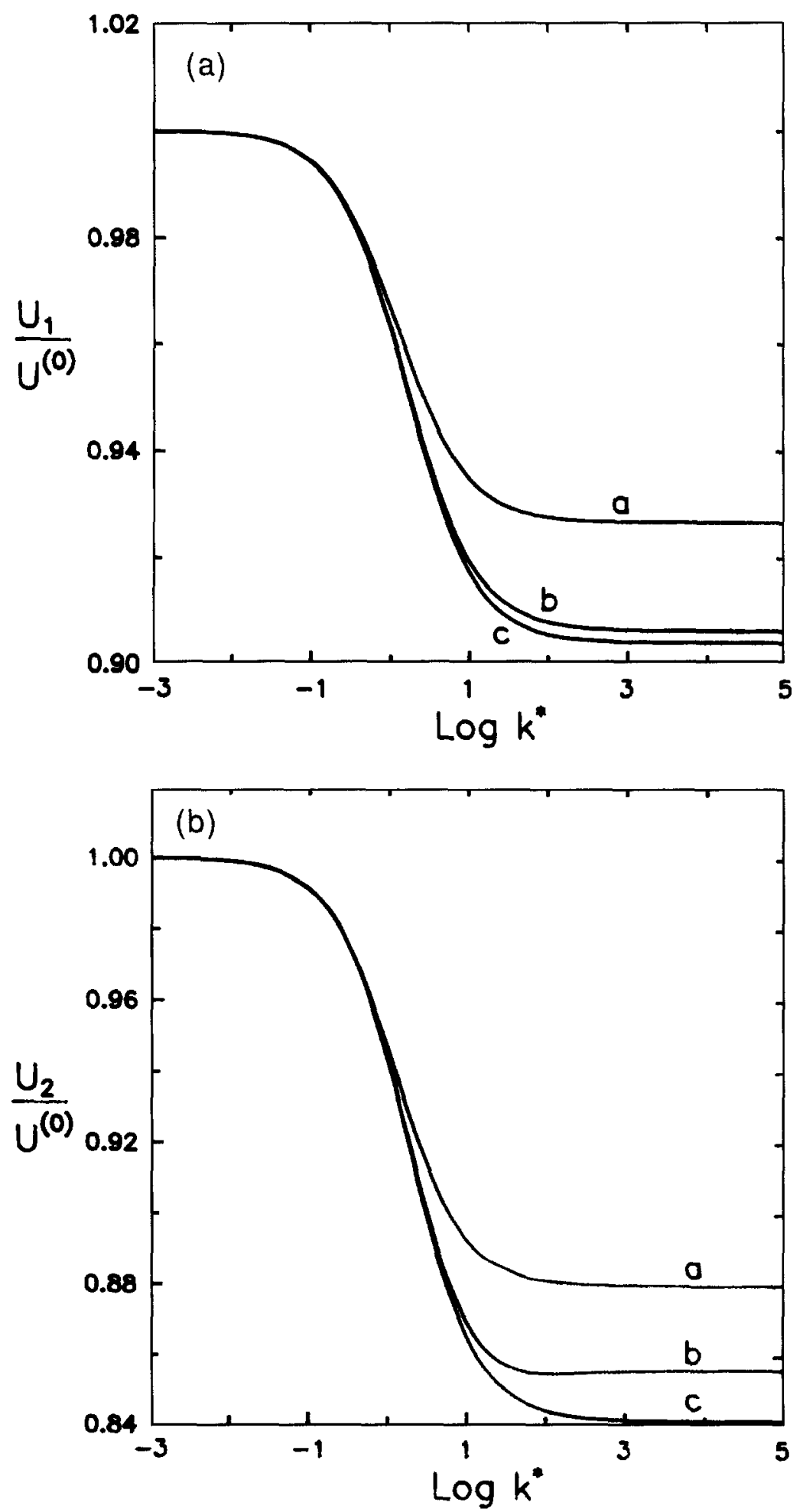

Fig. 4(a) and (b). 


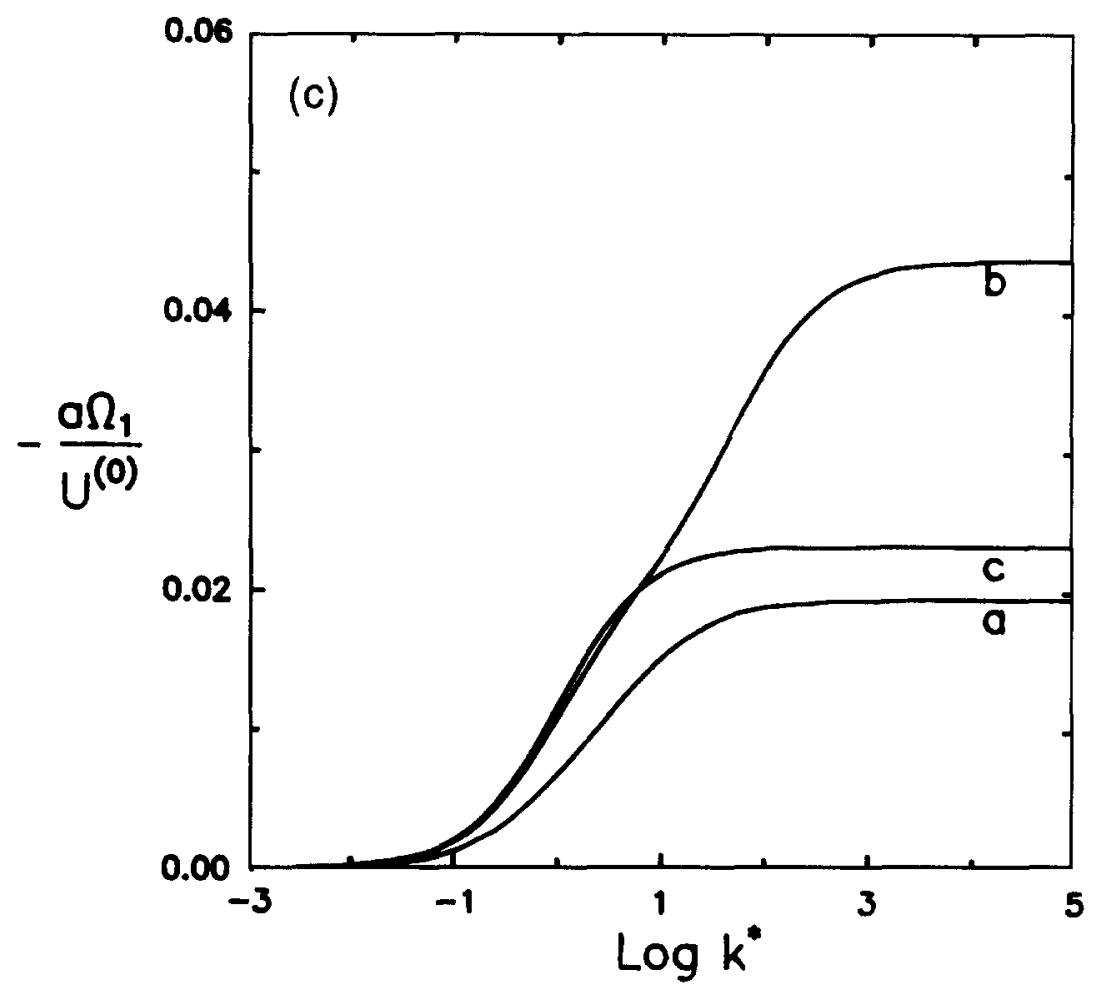

Fig. 4. Plots of the normalized translational and rotational velocities of three identical coaxial spheres of radius $a$ with equal spacings undergoing thermophoresis perpendicular to the line of their centers versus the thermal conductivity ratio $k^{*}$ with $C_{\mathrm{t}} l / a$ and $C_{\mathrm{m}} l / a$ as parameters $\left(2 a / r_{12}=0.8\right)$ :

(a) translational velocity of particle 1 (or particle 3 ); (b) translational velocity of particle 2; (c) rotational velocity of particle 1 (or particle 3 ). For curve (a), $C_{\mathrm{t}} l / a=0.2$ and $C_{\mathrm{m}} l / a=0.1$; for curve (b), $C_{\mathrm{l}} l / a=0.02$ and $C_{\mathrm{m}} l / a=0.01$; for curve (c), $C_{\mathrm{t}} l / a=C_{\mathrm{m}} l / a=0$.

connected by an infinitesimally thin and rigid rod. For this case, equation (31) can still be used to describe the translational and rotational velocities of two spheres; but now, the mobility parameters to account for the two-sphere interactions must reconcile with the relations given by equations (25) and (26).

Applying equation (26) for the two spheres and eliminating ${\underset{\sim}{0}}_{0}$, one has

$$
\underline{\sim}_{2}={\underset{\sim}{U}}_{1}+\underset{\sim}{\Omega} \times r_{12} \underset{\sim}{e} .
$$

Using the fact that $\underline{\sim}_{1}={\underset{\sim}{2}}_{2}=\Omega$ and substituting equation (31) into equation (33) yield

$$
\begin{gathered}
M_{11}^{(p)}=M_{21}^{(p)}, \\
M_{12}^{(p)}=M_{22}^{(p)}, \\
M_{11}^{(n)}=M_{21}^{(n)}+\frac{r_{12}}{a_{1}} N_{11}, \\
M_{12}^{(n)}=M_{22}^{(n)}+\frac{r_{12}}{a_{1}} N_{12}, \\
N_{11}=\frac{a_{1}}{a_{2}} N_{21}, \\
N_{12}=\frac{a_{1}}{a_{2}} N_{22} .
\end{gathered}
$$

Although the angular velocity $\Omega$ of the rigid dumbbell is independent of the location of its origin, the choice of the origin will affect the presentation of the results for the translational 
velocity ${\underset{\sim}{0}}_{0}$. Here we place the origin of the dumbbell at its center of hydrodynamic stress. The center of hydrodynamic stress is the unique point for bodies of revolution at which there is no coupling between translation and rotation (Happel and Brenner, 1983). For the dumbbell this point lies along the line connecting the centers of the spheres a distance $r_{01}$ from the center of sphere 1 . The detailed procedure to determine the ratio $r_{01} / r_{12}$ was provided by Fair and Anderson (1991) and its results for various values of $C_{\mathrm{m} 1} l / a_{1}, C_{\mathrm{m} 2} l / a_{2}$, $a_{2} / a_{1}$ and $\left(a_{1}+a_{2}\right) / r_{12}$ can be obtained by using the collocation method to solve for the hydrodynamic interactions between two aerosol spheres. Applying equation (26) for sphere 1 and using equation (33) to eliminate $\underset{\sim}{\Omega}$, one can express the translational velocity $\underline{\sim}_{0}$ of the dumbbell in terms of the translational velocities of the two spheres as

$$
\underline{\sim}_{0}=\left(1-\frac{r_{01}}{r_{12}}\right){\underset{\sim}{1}}_{1}+\frac{r_{01}}{r_{12}}{\underset{U}{2}}_{2}
$$

Substitution of equations (31a) and (34) into equation (35) leads to the expression

$$
\underline{\sim}_{0}=\sum_{j=1}^{2}\left[M_{i j}^{(p)} \underline{e} \underset{\sim}{e}+K_{i j}^{(n)}(\underset{\sim}{I}-\underline{e} \underline{e})\right] \cdot \underline{U}_{j}^{(0)},
$$

where $i$ can be either 1 or 2 , and

$$
\begin{aligned}
& K_{1 j}^{(n)}=M_{1 j}^{(n)}-\frac{r_{01}}{a_{1}} N_{1 j}, \\
& K_{2 j}^{(n)}=M_{2 j}^{(n)}+\frac{r_{02}}{a_{2}} N_{2 j} .
\end{aligned}
$$

Using equations (34c-f) and (37), one obtains

$$
\begin{aligned}
& K_{11}^{(n)}=K_{21}^{(n)}, \\
& K_{12}^{(n)}=K_{22}^{(n)} .
\end{aligned}
$$

In the limit $\left(a_{1}+a_{2}\right) / r_{12} \rightarrow 0$, it can be shown by the linearity of the Stokes equations that the dumbbell velocities

$$
\begin{gathered}
\underbrace{}_{0} \rightarrow \frac{a_{1} \hat{\lambda}_{1} \underline{U}_{1}^{(0)}+a_{2} \lambda_{2}{\underset{\sim}{2}}_{2}^{(0)}}{a_{1} \hat{\lambda}_{1}+a_{2} \lambda_{2}}, \\
\underline{\Omega} \rightarrow \frac{1}{r_{12}} \underset{e}{\operatorname{e}} \times\left[\underline{U}_{2}^{(0)}-\underline{\sim}_{1}^{(0)}\right],
\end{gathered}
$$

or the mobility parameters

where

$$
\begin{gathered}
M_{11}^{(p)}=K_{11}^{(n)}=\frac{r_{02}}{r_{12}}=\frac{a_{1} \lambda_{1}}{a_{1} \lambda_{1}+a_{2} \lambda_{2}}, \\
M_{12}^{(p)}=K_{12}^{(n)}=\frac{r_{01}}{r_{12}}=\frac{a_{2} \lambda_{2}}{a_{1} \lambda_{1}+a_{2} \lambda_{2}}, \\
N_{11}=-N_{12}=\frac{a_{1}}{r_{12}},
\end{gathered}
$$

$$
\lambda_{i}=\frac{1+2 C_{\mathrm{m} i} l / a_{i}}{1+3 C_{\mathrm{m} i} l / a_{i}}, \quad i=1 \text { or } 2 .
$$

In equation (39), $\underline{U}_{1}^{(0)}$ and $\underline{U}_{2}^{(0)}$ are the thermophoretic velocities of sphere 1 and sphere 2 , respectively, in the absence of the other, as given by equation (23).

To evaluate the mobility parameters $M_{11}^{(p)}$ and $M_{12}^{(p)}$, the axisymmetric thermophoresis of the dumbbell along the line connecting the sphere centers must be considered. For this case, 
the authors have derived the expression for the drag force exerted on each sphere in terms of the velocities of the spheres in a previous article (Chen and Keh, 1996). Using the fact that

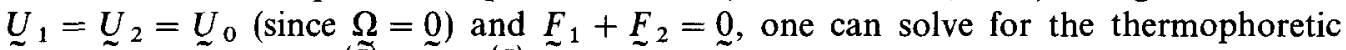
velocity (and hence, $M_{11}^{(\tilde{p})}$ and $M_{12}^{(p)}$ ) of the dumbbell. On the other hand, the translational and angular velocities of the dumbbell undergoing thermophoresis perpendicular to the line through the sphere centers need to be obtained to compute the mobility parameters $M_{11}^{(n)}$ (or $\left.K_{11}^{(n)}\right), M_{12}^{(n)}$ (or $\left.K_{12}^{(n)}\right), N_{11}$ and $N_{12}$. Similarly to the previous sections, equations (8) and (16) can be used to determine the unknown coefficients for the temperature and fluid velocity distributions in this case. However, equations (29) and (30), instead of equation (21), should be employed to solve the dumbbell velocities.

We have calculated the translational and angular velocities of a dumbbell in prescribed temperature gradients parallel and normal to the line of its sphere centers for various cases using the same numerical procedure presented in the previous sections. The results of the mobility parameters $M_{11}^{(p)}, M_{12}^{(p)}, M_{11}^{(n)}, M_{12}^{(n)}, N_{11}, N_{12}, K_{11}^{(n)}$ and $K_{12}^{(n)}$ and the ratio $r_{01} / r_{12}$ for a case of spheres having the same physical properties with various values of $a_{2} / a_{1}$ and $\left(a_{1}+a_{2}\right) / r_{12}$ are given in Table 4 . For the simplest case of the thermophoretic motion of a dumbbell having two identical spheres, the center of hydrodynamic stress of the dumbbell is in the middle of the connecting rod $\left(r_{01} / r_{12}=0.5\right)$, and the dumbbell will move without rotation $\left(N_{11}+N_{12}=0\right.$ or $\left.\underline{\Omega}=\underset{\sim}{0}\right)$. The value of $M_{11}^{(p)}+M_{12}^{(p)}$ (equal to the translational velocity of the dumbbell normalized by the undisturbed velocity of either of its two spheres, $U_{0} / U_{1}^{(0)}$, when the temperature gradient is prescribed along the connecting rod) is the same as that for the situation in which these two spheres are suspended freely and are separated by the same distance (Chen and Keh, 1996). However, the value of $M_{11}^{(n)}+M_{12}^{(n)}$ (equal to $K_{11}^{(n)}+K_{12}^{(n)}$ or $U_{0} / U_{1}^{(0)}$ of the dumbbell when the temperature gradient is applied normal to the connecting rod) is slightly smaller than that for the situation of freely-suspended spheres, as can be found in the comparison of Tables 1 and 4 . Note that the value of $M_{11}^{(n)}+M_{12}^{(n)}$ for

Table 4. The mobility parameters $M_{1 j}^{(p)}, M_{1 j}^{(n)}, N_{1 j}$ and $K_{1 j}^{(n)}$ and the ratio $r_{01} / r_{12}$ for the thermophoresis of a rigid dumbbell composed of two spheres having the same physical properties with $k^{*}=100, C_{1} l / a_{1}=0.2$ and $C_{\mathrm{m}} l / a_{1}=0.1$

\begin{tabular}{|c|c|c|c|c|c|c|c|c|c|c|}
\hline$\frac{a_{2}}{a_{1}}$ & $\frac{a_{1}+a_{2}}{r_{12}}$ & $M_{11}^{(p)}$ & $M_{12}^{(p)}$ & $M_{11}^{(n)}$ & $M_{12}^{(n)}$ & $-N_{11}$ & $N_{12}$ & $K_{11}^{(n)}$ & $K_{12}^{(n)}$ & $\frac{r_{01}}{r_{12}}$ \\
\hline \multirow[t]{9}{*}{1} & 0.0 & 0.5000 & 0.5000 & 0.0000 & 1.0000 & 0.0000 & 0.0000 & 0.5000 & 0.5000 & 0.5000 \\
\hline & 0.2 & 0.5011 & 0.5011 & 0.0089 & 0.9900 & 0.0981 & 0.0981 & 0.4995 & 0.4995 & 0.5000 \\
\hline & 0.4 & 0.5081 & 0.5081 & 0.0263 & 0.9652 & 0.1878 & 0.1878 & 0.4958 & 0.4958 & 0.5000 \\
\hline & 0.6 & 0.5241 & 0.5241 & 0.0397 & 0.9324 & 0.2678 & 0.2678 & 0.4861 & 0.4861 & 0.5000 \\
\hline & 0.8 & 0.5454 & 0.5454 & 0.0390 & 0.8980 & 0.3436 & 0.3436 & 0.4685 & 0.4685 & 0.5000 \\
\hline & 0.9 & 0.5551 & 0.5551 & 0.0307 & 0.8838 & 0.3839 & 0.3839 & 0.4572 & 0.4572 & 0.5000 \\
\hline & 0.95 & 0.5591 & 0.5591 & 0.0239 & 0.8791 & 0.4062 & 0.4062 & 0.4515 & 0.4515 & 0.5000 \\
\hline & 0.99 & 0.5616 & 0.5616 & 0.0170 & 0.8773 & 0.4258 & 0.4258 & 0.4472 & 0.4472 & 0.5000 \\
\hline & 1.0 & 0.5621 & 0.5621 & 0.0146 & 0.8772 & 0.4310 & 0.4310 & 0.4462 & 0.4462 & 0.5000 \\
\hline \multirow[t]{9}{*}{2} & 0.0 & 0.3255 & 0.6745 & -0.0000 & 2.0725 & 0.0000 & 0.0000 & 0.3255 & 0.6745 & 0.6745 \\
\hline & 0.2 & 0.3022 & 0.7000 & -0.0384 & 2.0361 & 0.0341 & 0.1311 & 0.3124 & 0.6873 & 0.6859 \\
\hline & 0.4 & 0.2798 & 0.7358 & -0.0615 & 1.9671 & 0.0676 & 0.2409 & 0.2924 & 0.7060 & 0.6980 \\
\hline & 0.6 & 0.2619 & 0.7817 & -0.0819 & 1.9104 & 0.0963 & 0.3318 & 0.2601 & 0.7320 & 0.7103 \\
\hline & 0.8 & 0.2459 & 0.8300 & -0.1088 & 1.8893 & 0.1199 & 0.4155 & 0.2162 & 0.7631 & 0.7228 \\
\hline & 0.9 & 0.2367 & 0.8510 & -0.1246 & 1.9016 & 0.1300 & 0.4611 & 0.1915 & 0.7805 & 0.7294 \\
\hline & 0.95 & 0.2314 & 0.8596 & -0.1318 & 1.9196 & 0.1345 & 0.4879 & 0.1794 & 0.7905 & 0.7328 \\
\hline & 0.99 & 0.2267 & 0.8652 & -0.1345 & 1.9457 & 0.1371 & 0.5141 & 0.1711 & 0.7997 & 0.7356 \\
\hline & 1.0 & 0.2232 & 0.8712 & -0.1351 & 1.9583 & 0.1372 & 0.5229 & 0.1680 & 0.8033 & 0.7363 \\
\hline \multirow[t]{9}{*}{5} & 0.0 & 0.1584 & 0.8416 & -0.0000 & 5.3145 & 0.0000 & 0.0000 & 0.1584 & 0.8416 & 0.8416 \\
\hline & 0.2 & 0.1310 & 0.8707 & -0.0758 & 4.9161 & 0.0086 & 0.1581 & 0.1449 & 0.8594 & 0.8553 \\
\hline & 0.4 & 0.1055 & 0.9049 & -0.1038 & 4.1835 & 0.0174 & 0.2524 & 0.1231 & 0.8927 & 0.8692 \\
\hline & 0.6 & 0.0834 & 0.9405 & -0.1058 & 3.5639 & 0.0226 & 0.2973 & 0.0939 & 0.9366 & 0.8837 \\
\hline & 0.8 & 0.0620 & 0.9705 & -0.0979 & 3.1378 & 0.0234 & 0.3198 & 0.0600 & 0.9792 & 0.9000 \\
\hline & 0.9 & 0.0509 & 0.9808 & -0.0902 & 2.9906 & 0.0221 & 0.3290 & 0.0438 & 0.9951 & 0.9098 \\
\hline & 0.95 & 0.0452 & 0.9840 & -0.0839 & 2.9401 & 0.0209 & 0.3352 & 0.0369 & 1.0026 & 0.9152 \\
\hline & 0.99 & 0.0406 & 0.9852 & -0.0752 & 2.9257 & 0.0192 & 0.3440 & 0.0318 & 1.0080 & 0.9198 \\
\hline & 1.0 & 0.0389 & 0.9877 & -0.0701 & 2.9344 & 0.0187 & 0.3481 & 0.0332 & 1.0110 & 0.9209 \\
\hline
\end{tabular}



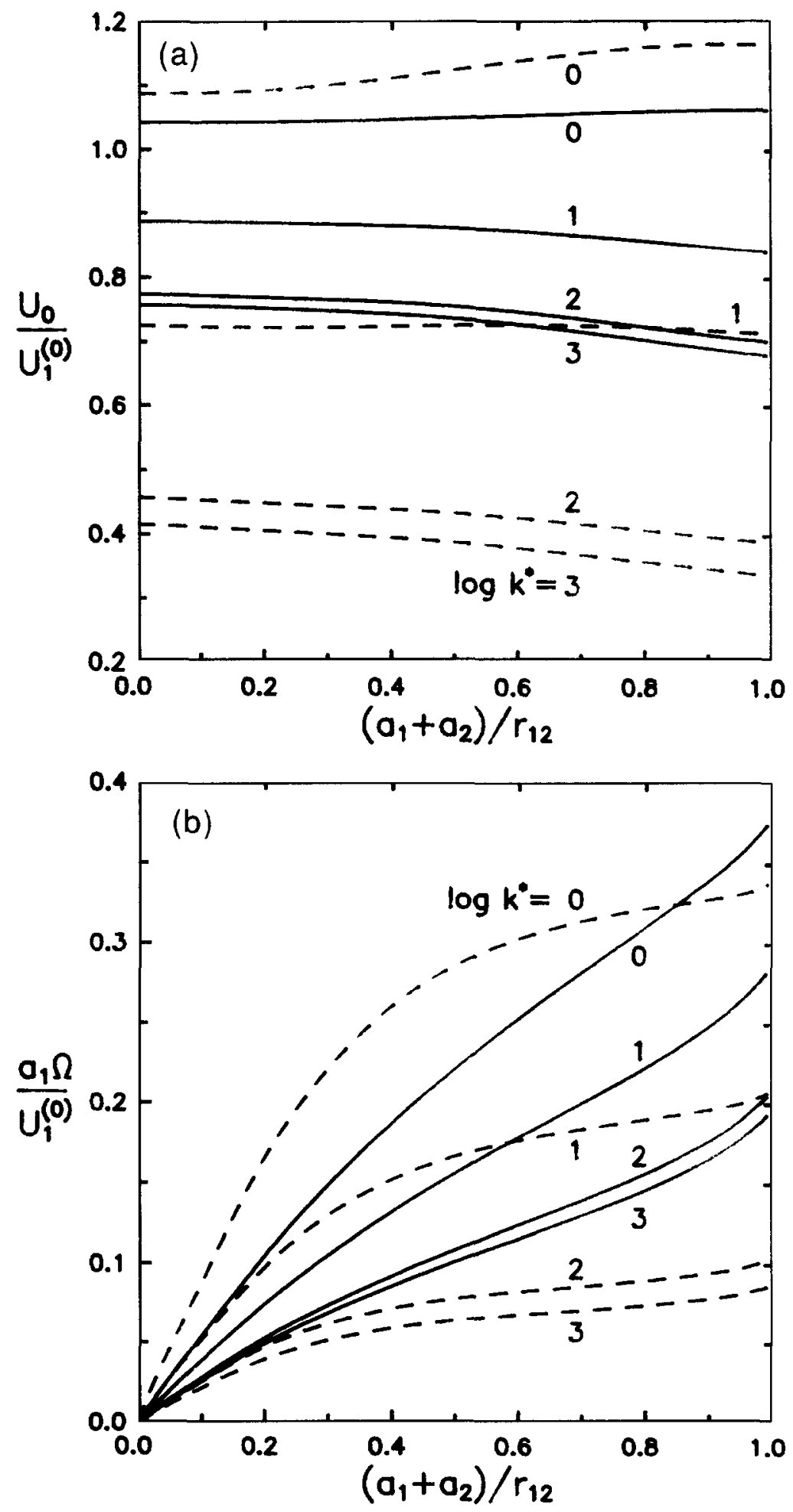

Fig. 5. Plots of the dimensionless translational and rotational velocities of a rigid dumbbell normal to the line connecting its two spheres versus the separation parameter with various values of $a_{2} / a_{1}$ and $k^{*}$. The solid curves are plotted for the case $a_{2} / a_{1}=2$, whereas the dashed curves are plotted for the case of $a_{2} / a_{1}=5$. In all cases, $C_{\mathrm{t}} l / a_{1}=0.2$ and $C_{\mathrm{m}} l / a_{1}=0.1$. 
the dumbbell decreases monotonically as the separation parameter $2 a / r_{12}$ increases, while this value for two freely suspended spheres is not a monotonic function of $2 a / r_{12}$.

Since flocculation of different-sized particles can occur in an aerosol, it might be important to investigate the thermophoretic motion of such a dumbbell. Results of the mobility parameters and $r_{01} / r_{12}$ for a dumbbell composed of two spheres with $a_{2} / a_{1}=2$ and 5 are also listed in Table 4. Examination of these data indicates that equation (40) is very well satisfied as $\left(a_{1}+a_{2}\right) / r_{12} \rightarrow 0$ for all cases considered. In a previous article (Chen and Keh, 1996), the normalized thermophoretic velocity of a dumbbell with $a_{2} / a_{1}=2$ and 5 along its connecting rod were plotted versus $\left(a_{1}+a_{2}\right) / r_{12}$ in a figure for various values of $k^{*}$. The corresponding plot for a dumbbell undergoing thermophoresis normal to its connecting rod is given in Fig. 5. It can be seen that the translational velocity of the thermophoretic dumbbell is not necessarily a monotonic function of $\left(a_{1}+a_{2}\right) / r_{12}$.

\section{CONCENTRATION DEPENDENCE OF THERMOPHORETIC VELOCITY}

In practical applications of thermophoresis, collections of aerosol particles in bounded systems are usually encountered. Therefore, it is necessary to determine the dependence of the mean thermophoretic mobility of an aerosol on particle concentration. Based on a microscopic model of particle interactions in a polydisperse dispersion which involves both statistical and low Reynolds number hydrodynamic concepts (Batchelor, 1972; Reed and Anderson, 1980), the mean thermophoretic velocity of type $i$ particles (having radii $a_{i}$ and physical properties $C_{\mathrm{s} i}, C_{t i}, C_{\mathrm{m} i}$ and $k_{i}^{*}$ ) in a bounded suspension of particles that have a distribution in both radius and physical properties can be expressed as (Keh and Chen, 1995)

$$
\left\langle\underline{U}_{i}\right\rangle=\underline{\sim}_{i}^{(0)}\left[1+\sum_{j} \alpha_{i j} \varphi_{j}+\mathrm{O}\left(\varphi^{2}\right)\right],
$$

where ${\underset{\sim}{U}}_{i}^{(0)}$ is defined in equation (23) and

$$
\begin{aligned}
\alpha_{i j}= & -\frac{A_{j}}{A_{i}}-\frac{1-k_{j}^{*}+k_{j}^{*} C_{\mathrm{t} j} l / a_{j}}{2+k_{j}^{*}+2 k_{j}^{*} C_{\mathrm{t} j} l / a_{j}} \\
& +\left(1+\frac{a_{i}}{a_{j}}\right)^{3} \int_{0}^{1}\left\{\left[M_{11}^{(p)}+2 M_{11}^{(n)}-3\right]+\frac{A_{j}}{A_{i}}\left[M_{12}^{(p)}+2 M_{12}^{(n)}\right]\right\} w^{-4} \mathrm{~d} w
\end{aligned}
$$

with

$$
w=\frac{a_{1}+a_{2}}{r_{12}} \quad\left(a_{1}=a_{i} \text { and } a_{2}=a_{j}\right)
$$

Here, $\varphi_{j}$ is the volume fraction of type $j$ particles (having radius $a_{j}$ and physical properties $C_{\mathrm{tj}}, C_{\mathrm{m} j}, C_{\mathrm{s} j}$ and $\left.k_{j}^{*}\right)$ in the aerosol, $\varphi$ is the volume fraction of all particles $\left(=\sum_{j} \varphi_{j}\right)$, and $M_{11}^{(p)}, M_{12}^{(p)}, M_{11}^{(n)}$ and $M_{12}^{(n)}$ are the mobility parameters defined by equation (31a) for two-sphere thermophoresis. For a dispersion of identical spheres (monodisperse system), equation (42) for the mean thermophoretic velocity reduces to

$$
\langle\underline{U}\rangle=\underline{U}^{(0)}\left[1+\alpha \varphi+\mathrm{O}\left(\varphi^{2}\right)\right]
$$

where coefficient $\alpha$ equals the value of $\alpha_{i j}$ with $a_{i}=a_{j}, C_{\mathrm{t} i}=C_{\mathrm{t} j}, C_{\mathrm{m} i}=C_{\mathrm{m} j}, k_{i}=k_{j}$ and $A_{i}=A_{j}$.

In deriving equation (43), it has been assumed that the particles are hard spheres without long-range pair potential and the two-particle radial distribution function has its equilibrium values. The mean particle velocities are calculated for a reference frame in which the net particle and fluid flux is zero and the volume average of the temperature gradient field over a representative sample of the suspension is $\underset{\sim}{E_{\infty}}$. Note that equations (42) and (45) are valid for a bounded system when the cloud of particles is far from any walls. 
Table 5. The results of coefficients $\alpha_{i j}$ for a suspension of aerosol spheres having the same physical properties with $C_{1} l / a_{i}=0.2$ and $C_{\mathrm{m}} l / a_{i}=0.1$ at various values of $k^{*}$ and $a_{j} / a_{i}$

\begin{tabular}{cccc}
\hline & & \multicolumn{2}{c}{$-\alpha_{i j}$} \\
\cline { 3 - 3 }$a_{i}$ & $k^{*}$ & $\begin{array}{l}\text { Collocation } \\
\text { technique }\end{array}$ & $\begin{array}{l}\text { Method of } \\
\text { reflections }\end{array}$ \\
\hline 0.5 & 1000 & 0.886 & 2.144 \\
& 100 & 0.864 & 2.095 \\
& 10 & 0.780 & 1.794 \\
1 & 0.848 & 1.476 \\
& 0.1 & 1.062 & 1.429 \\
& 0.01 & 1.071 & 1.412 \\
& 1000 & 0.418 & 0.901 \\
& 100 & 0.430 & 0.905 \\
& 10 & 0.528 & 0.960 \\
& 1 & 0.810 & 1.287 \\
& 0.1 & 1.187 & 1.475 \\
& 0.01 & 1.251 & 1.498 \\
& 1000 & -0.108 & 0.010 \\
& 100 & -0.064 & 0.063 \\
& 10 & 0.258 & 0.374 \\
& 1 & 0.978 & 1.168 \\
& 0.1 & 1.371 & 1.530 \\
& 0.01 & 1.431 & 1.579 \\
\hline
\end{tabular}

The collocation results of $M_{11}^{(n)}$ and $M_{12}^{(n)}$ as a function of $w$ for two spheres having the same physical properties with various values of $C_{\mathrm{t}} l / a_{1}, C_{\mathrm{m}} l / a_{1}, k^{*}$ and $a_{2} / a_{1}$ were presented in Section 3, while the corresponding numerical solutions of $M_{11}^{(p)}$ and $M_{12}^{(p)}$ were obtained in previous studies (Chen and Keh, 1995, 1996). Thus, the integration in equation (43) can be performed numerically using these data, and the results of the interaction coefficient $\alpha_{i j}$ for a suspension of aerosol particles of the same material at various value of $k^{*}$ and $a_{j} / a_{i}$ are listed in Table 5 for the case of $C_{\mathrm{t}} l / a_{i}=0.2$ and $C_{\mathrm{m}} l / a_{i}=0.1$. The corresponding results of $\alpha_{i j}$, computed from the analytical formula derived by using the method of reflections (Keh and Chen, 1995), are also listed in the last column of the same table for comparison. It shows that the approximate results of $\alpha_{i j}$ obtained from the method of reflections always overestimate its exact values and the error can be quite significant. In general cases (and in monodisperse systems), coefficient $\alpha_{i j}$ is negative and the mean thermophoretic velocity in an aerosol will be smaller when the volume fraction of the particles is increased. However, the value of $\alpha_{i j}$ can become positive if both the values of $k^{*}$ and $a_{j} / a_{i}$ are large.

\section{CONCLUDING REMARKS}

In this work the thermophoresis of a finite assemblage of aerosol spheres in an arbitrary configuration is studied by a combined analytical-numerical method. The spheres may differ in size and in physical properties. Not only the particle interactions among free spheres but also the movement of a rigid cluster of connected spheres has been examined. A boundary-collocation technique has been used to obtain the temperature distribution inside and outside the spheres and the velocity field for the surrounding fluid. The results for the particle-interaction parameters indicate that the solution procedure converges rapidly and solutions can be obtained to the satisfactory degree of accuracy for various cases even when the spheres are touching one another.

In Section 2, the linear algebraic formulas to solve the general problem of thermophoretic motion of multiple spheres were derived and the procedure of the application of the collocation technique was given. The numerical results to correct equation (1) for systems of two free spheres, of three free spheres, and of a rigid cluster of two spheres were obtained for various cases in Sections 3-5. Due to the fact that the number of relevant variables in the 
general problem of multiple spheres is very great, we did not present the results for systems having more than three spheres. However, the solution technique used in this work can also provide the numerical calculations for the thermophoresis of four or more spheres. In a similar problem for the motion of particles driven by gravity, results of particle interactions were obtained using the same collocation method for three-dimensional clusters of up to 64 spheres (Hassonjee et al., 1988). In Section 6, the collocation solutions for the interaction between two spheres were used to calculate the mean thermophoretic velocity in a bounded suspension of aerosol spheres.

\section{REFERENCES}

Batchelor, G. K. (1972) Sedimentation in dilute dispersion of spheres. J. Fluid Mech. 52, 245-268.

Brock, J. R. (1962) On the theory of thermal forces acting on aerosol particles. J. Colloid Sci. 17, 768-780.

Chen, S. H. and Keh, H. J. (1995) Axisymmetric thermophoretic motion of two spheres. J. Aerosol Sci. 26, 429-444.

Chen, S. H. and Keh, H. J. (1996) Axisymmetric thermophoresis of multiple aerosol spheres. Aerosol Sci. Technol. 24, 21-35.

Fair, M. C. and Anderson, J. L. (1990) Electrophoresis of dumbbell-like colloid particles. Int. J. Multiphase Flow 16, 663-679.

Friedlander, S. K. (1977) Smoke, Dust and Haze, pp. 42-44. Wiley, New York.

Happel, J. and Brenner, H. (1983) Low Reynolds Number Hydrodynamics. Martinus Nijhoff, The Netherlands.

Hassonjee, Q., Ganatos, P. and Pfeffer, R. (1988) A strong-interaction theory for the motion of arbitrary three-dimensional clusters of spherical particles at low Reynolds number. J. Fluid Mech. 197, 1-37.

Keh, H. J. and Chen, S. H. (1995) Particle interactions in thermophoresis. Chem. Engng Sci. 50, 3395-3407.

Keh, H. J. and Yang, F. R. (1991) Particle interactions in electrophoresis. IV. Motion of arbitrary threedimensional clusters of spheres. J. Colloid Inter. Sci. 145, 362-389.

Kennard, E. H. (1938) Kinetic Theory of Gases. McGraw-Hill, New York.

Leong, K. H. (1984) Thermophoresis and diffusiophoresis of large aerosol particles of different shapes. J. Aerosol Sci. 15, 511-517.

Montassier, N., Boulaud, D. and Renoux, A. (1991) Experimental study of thermophoretic particle deposition in laminar tube flow. J. Aerosol Sci. 22, 677-687.

Reed, C. C. and Anderson, J. L. (1980) Hindered settling of a suspension at low Reynolds number. A.I.Ch.E. J. 26, $816-827$.

Sasse, A. G. B. M., Nazaroff, W. W. and Gadgil, A. J. (1994) Particle filter based on thermophoretic deposition from nature convection flow. Aerosol Sci. Technol. 20, 227-238.

Talbot, L., Cheng, R. K., Schefer, R. W. and Willis, D. R. (1980) Thermophoresis of particles in heated boundary layer. J. Fluid. Mech. 101, 737-758.

Waldmann, L. and Schmitt, K. H. (1966) Thermophoresis and diffusiophoresis of aerosols. In Aerosol Science (Edited by Davies, C. N.). Academic Press, New York.

Weinberg, M. C. (1982) Thermophoretic efficiency in modified chemical vapor deposition process. J. Am. Ceram. Soc. 65, 81-87.

Williams, M. M. R. and Loyalka, S. K. (1991) Aerosol Science: Theory and Practice, with Special Applications to the Nuclear Industry. Pergamon Press, Oxford.

Ye, Y., Pui, D. Y. H., Liu, B. Y. H., Opiolka, S., Blumhorst, S. and Fissan, H. (1991) Thermophoretic effect of particle deposition on a free standing semiconductor wafer in a clean room. J. Aerosol Sci. 22, 63-72. 